\title{
Uusioersily
}

\section{Evolving, dynamic clustering of spatio/spectro-temporal data in 3D spiking neural network models and a case study on EEG data}

Doborjeh, M., Kasabov, N., \& Doborjeh, Z. (2017). Evolving, dynamic clustering of spatio/spectro-temporal data in 3D spiking neural network models and a case study on EEG data. Evolving Systems, 195-211. [9178]. https://doi.org/10.1007/s12530-017-9178-8

Link to publication record in Ulster University Research Portal

\author{
Published in: \\ Evolving Systems
}

Publication Status:

Published (in print/issue): 17/04/2017

DOI:

$10.1007 / \mathrm{s} 12530-017-9178-8$

\section{Document Version}

Author Accepted version

\section{General rights}

Copyright for the publications made accessible via Ulster University's Research Portal is retained by the author(s) and / or other copyright owners and it is a condition of accessing these publications that users recognise and abide by the legal requirements associated with these rights.

\section{Take down policy}

The Research Portal is Ulster University's institutional repository that provides access to Ulster's research outputs. Every effort has been made to ensure that content in the Research Portal does not infringe any person's rights, or applicable UK laws. If you discover content in the Research Portal that you believe breaches copyright or violates any law, please contact pure-support@ulster.ac.uk. 


\section{Evolving, dynamic clustering of spatio/spectro-temporal data

5 Maryam Gholami Doborjeh $^{1} \cdot$ Nikola Kasabov $^{1} \cdot$ Zohreh Gholami Doborjeh $^{1}$

Received: 12 June 2016 / Accepted: 14 February 2017

(C) Springer-Verlag Berlin Heidelberg 2017
EEG data, recorded from three groups of subjects during a cognitive task. The clusters were referred back to the brain data for a better understanding of the data and the processes that generated it. The cluster analysis allowed to discover and understand differences on temporal sequences and spatial involvement of brain regions in response to a cognitive task.

Keywords Dynamic spatiotemporal streaming data clustering $\cdot$ EEG data $\cdot$ NeuCube $\cdot$ Spiking neural networks · Unsupervised learning · Supervised learning · Personalised clustering

\section{Introduction}

Clustering aims at objectively organise data samples into homogenised groups, where the data samples within a group are similar. So far many clustering methods have been developed to identify structures in different data types, such as static, temporal, etc. Data is static when the feature values do not change over time, and it is time series (temporal) if the features change their values over a continuous time. With respect to different data types, clustering methods differ significantly in the notion of the similarity or distance measures.

Clustering can be classified according to several criteria:

i. Clustering of raw data versus clustering of pre-processed data (such as encoded into spikes, as the case in the paper is);

ii. Clustering of absolute values of variables versus clustering of changes in the variables (also called events, as the case in this paper is); and School of Engineering, Computer and Mathematical Sciences, Auckland University of Technology, Auckland, New Zealand 
iii. Direct clustering of data versus clustering of a model that is being created to learn and capture the essential characteristics of interest from the data (as the case in this paper is);

iv. Clustering of vector based, static data versus clustering of dynamic, streaming data (as the case in the paper is).

We can distinguish the following classes of clustering methods for static, vector based data:

a) Clustering of static, vector based data, where the number of clusters is pre-defined (such as C-means, K-Mean clustering methods);

b) Clustering of time series data, where the number of the time points are fixed and the time series samples are represented as vectors, applying the above methods.

c) Evolving clustering methods, where a stream of vectors are clustered incrementally without pre-defining the number of clusters such as DENFIS (Kasabov and Song 2002) and Evolving Fuzzy Neural Network (EFuNN) (Kasabov 2001);

In this paper, we propose clustering methods for whole spatiotemporal data samples from a data stream. In principle, these methods can be classified into the following groups:

d) Two tier clustering of the data: first according to the spatial information, and then according to the temporal information;

e) Integrated clustering of both spatial and temporal information;

f) In case of both static and spatiotemporal data are available, clustering of the static information first, and then-the spatiotemporal information. This can be applied for personalised modelling(Gholami Doborjeh and Kasabov 2016).

g) Integrated clustering of both static and spatiotemporal data for personalised modelling.

The paper presents two methods in the groups (d) and (e) from above that are also defined in the categories (i)-(iv).

\subsection{Overview on static data clustering, temporal data clustering and evolving clustering methods}

Clustering methods for various static data are classified into five major categories: hierarchical methods (Johnson 1967); partitioning methods; density-based methods (Ester et al. 1996); grid-based methods; and model-based methods. Partitioning methods construct $k$ partitions of data, where each partition is represented by a similarity metric of the objects in the partition. A partition is hard if each object belongs to only one cluster [k-means (Hartigan and Wong 1979)], or fuzzy if one object is allowed to be in more than one cluster by different degree [fuzzy c-means (Bezdek et al. 1984)]. A hierarchical clustering represents groups of data objects using a tree of clusters, where the similarity is measured using a pairwise distance matrix of the objects. In density-based clustering, a cluster is continuously growing as long as the density in the neighbourhood surpasses a threshold. In grid-based clustering, the object space is quantized into a finite number of cells, where the operations for clustering are performed. A common example of the grid-based approach is STING (Wang et al. 1997). Model-based clustering undertakes a model for each cluster and aims at best fit of data to the model. One major method of model-based clustering is neural network approach. Two prominent clustering methods of the neural network are competitive learning, including ART (Carpenter and Grossberg 1987) and self-organizing maps (SOM) (Kohonen 1998).

Massive amount of temporal data (time-series data) has been recorded so far in various areas, such as electronic, video/audio, biologic, neurology, etc. In case of clustering of such data, given a set of individual time series values, the objective is to group similar patterns into the same cluster. This task demands a measure notion to estimate the level of similarity between time series. However, the known Euclidean distance and other typical measures used for non-temporal data are unsuitable metrics to evaluate the similarity between time series, because they are unable to deal with temporal interaction between time series data features. Various temporal data clustering methods have been introduced so far, such as biclustering (Mirkin 1998) that has been used for clustering the time series gene expression data (Tanay et al. 2002). Any data that can be represented as a matrix is amenable to biclustering. Biclustering methods simultaneously clusters both rows and columns of a matrix.

Evolving clustering methods represent incremental growth of clusters and creation of new clusters from a stream of vector based data. While SOM assigns similar input vectors into topologically close neurons, evolving Self-organizing maps (ESOM) (Deng and Kasabov 2000) and the DENFIS evolving clustering method (Kasabov and Song 2002) were introduced for online unsupervised data clustering. EFuNN (Kasabov 2001) was introduced for evolving supervised clustering related to classification or regression. In (Katwal et al. 2013), a graph-based visualisations of SOM has been used for clustering fMRI data. In another research (Liao et al. 2008), an integrated SOM and hierarchical clustering architecture was designed to detect activation patterns of fMRI data. While using these methods for clustering of time series data, the temporal components of each sample data variables are transposed into 
feature vectors, where the time is hidden and no temporal interaction of time series within a sample can be learned anymore.

A method of spatiotemporal clustering has been proposed in (Deng et al. 2013) for clustering input variables of meteorological data. In this research the input variables can be clustered if they are within a certain neighbourhood and their dynamics are auto-correlated.

All the above methods deal with vector based data and do not reveal any spatiotemporal information related to the processes that generated the data. This is a significant contrast to the clustering methods that we propose in this paper as they deal with whole spatiotemporal patterns of data.

Unlike the above methods that aimed to directly cluster raw data, we propose to dynamically cluster not the raw data, but the changes in the data that are dynamically captured in a brain-like evolving SNN computational model. The model is dynamically evolving from the input spatiotemporal data after the data is encoded into spikes representing changes in the data, and the clusters in the model are evolving too. These clusters represent: (1) dynamic measures of spatiotemporal similarity in the input variables in respect to their changing values in time; (2) sequentially activated areas of the SNN model that capture changes in the data over time.

\subsection{Spatiotemporal data clustering}

Spatiotemporal clustering is a procedure of grouping data samples based on their spatial and temporal similarity. Learning dynamic patterns of spatiotemporal data is a challenging task, as temporal features may manifest complex interaction that may also change dynamically over time. Developing new clustering methods that can capture these "hidden" interactions and interrelationships among multivariate data, is of crucial importance not only for the spatiotemporal data analysis and data understanding, but also for future events prediction based on captured complex spatiotemporal patterns from Spatio-Spectro-Temporal Data (SSTD). A variety of techniques have been developed for SSTD recordings, such as EEG, fMRI, DTI, etc. (Niedermeyer and da Silva 2005; Ogawa et al. 1992).

SNN methods have been developed for SSTD learning, some of them are implemented in neuromorphic hardware systems (Indiveri et al. 2011). The challenge now for information science and artificial intelligence is to develop new computational methods that utilize SNN and neuromorphic hardware systems for efficient processing of SSTD, including clustering of such data.

NeuCube (Kasabov 2014) is a generic evolving SpatioTemporal Data Machine (STDM) based on SNN for learning, classification/regression, visualisation and interpretation of spatiotemporal data. It is able to capture both time and space features of SSTD in a SNN architecture for the sake of understanding the data. When compared with traditional statistical analysis methods and methods of artificial intelligence when dealing with SSTD, NeuCube models resulted in significantly higher accuracy of classification results, faster data processing, and a better visualisation and interpretation of the SSTD. This is due to the ability of a NeuCube model to learn and capture spatiotemporal interaction between the data variables. So far NeuCube has been successfully used for EEG data modelling, learning, and classification (Gholami Doborjeh et al. 2016; Kasabov and Capecci 2015; Capecci et al. 2015; Schliebs et al. 2013).

This paper now contributes to the NeuCube computational framework with dynamic clustering of spatiotemporal connectivity and spiking activity of the spiking neurons in a NeuCube model, while it is learning from streaming data.

This paper introduces new methods for dynamic, evolving clustering of spatiotemporal streaming data through encoding the data into spikes, creating dynamic, evolving clusters of spiking neurons in a 3D SNN reservoir, such as the NeuCube system, both in space and time. The main purposes of the introduced methods, that have not been achieved so far, are the following:

1. To detect similar spatiotemporal patterns of changes in the streaming data, which are dynamically generated with respect to the interaction between input variables. The dynamic visualisation of the clusters captures the time in which a cluster is created and it demonstrates how this cluster is changed over time. It enables us for the first time to study the dynamics of such clusters.

2. To understand hidden spatiotemporal patterns of changes in the data by pursuing the trend of the cluster creation. For the case study shown in the paper, this relates to brain activities.

The clustering methods are applied to a case study of EEG data, but can be used for other spatiotemporal data, such as fMRI (Gholami Doborjeh and Kasabov 2015), seismic and other data (Kasabov et al. 2016).

This paper is structured as follows: Sect. 2 describes the NeuCube architecture for SSTD analysis (Kasabov 2014). Section 3 introduces the proposed methods for clustering of SSTD in an unsupervised and supervised learning modes. Section 4 describes the case study data. Section 5 describes the details of the proposed dynamic unsupervised clustering methods and illustrates them on the case study data. Section 6 describes the details of the personalised clustering methods at supervised learning stage and illustrates them on the case study data. Section 7 represents a comparison of the proposed clustering methods with SOM clustering. Section 8 discusses results and future directions.

\begin{tabular}{|l|l|l|l|l|}
\hline Journal : Large 12530 & Article No : 9178 & Pages : 17 & MS Code : EVOS-D-16-00033 & Dispatch : 27-3-2017 \\
\hline
\end{tabular}




\section{The NeuCube spiking neural network architecture for SSTD}

Spiking Neural Network (SNN) models can learn both space and time components from data and are considered as suitable models to process SSTD (Maass et al. 2002). The NeuCube-based SNN architecture (shown graphically in Fig. 1) consists of: input data encoding module; that encodes multivariable continuous temporal stream data into spike trains; a 3D recurrent SNN cube (SNNcube), where input data are mapped and learned in an unsupervised mode; and an SNN classifier that learns in a supervised mode to classify the spatiotemporal patterns of the SNNcube activities which represent patterns from the input data (Kasabov 2007, 2012, 2014; Kasabov et al. 2013).

\subsection{Input data encoding, mapping, and model initialisation}

\subsubsection{Input spatiotemporal data encoding}

In one of the implementations of the NeuCube encoding module, a Threshold-Based Representation method (TBR) (Delbruck 2007) is applied to the SSTD to produce spike trains. Generated spikes represent the form of the SSTD wave signal in terms of amplitude changes. Once a signal change exceeds the threshold $T B R_{t h r}$, one spike occurs. Therefore, in the case of brain data, the spike trains represent real brain activity patterns that will be used to train a SNNcube.

\subsubsection{SSTD mapping to the SNNcube}

A 3D brain-like SNN cube is created to map a relevant brain data template, such as Talairach (Talairach and Tournoux 1988), MNI (Lancaster et al. 2007), etc. The size of the SNNcube is controlled by three parameters: $n_{x}, n_{y}, n_{z}$ representing the number of spiking neurons along $x, y$ and $z$ coordinates. Every neuron in this cube is a computational unit that is implemented based on the Leaky-Integrate and Fire Model (LIFM) of a spiking neuron as one implementation (Abbott 1999). Input neurons are allocated to the input SSTD variables for transferring their spike trains to the SNNcube. In order to preserve the spatial information of the SSTD, each neuron in the SNNcube represents a spatial location from the brain template, therefore - an area of the brain. Also each allocated input neuron in the SNNcube has the same $(x, y, z)$ coordinates as the corresponding input data variable in the used brain template.

\subsubsection{SNNcube initialisation}

The SNNcube is initialised with the use of the "small world" connectivity. Each neuron in the SNNcube is connected to its nearby neurons, which are within a maximum distance $d$. The initial connection weight between neurons $i$ and $j$, denoted by $w_{i, j}$, is defined as follow:

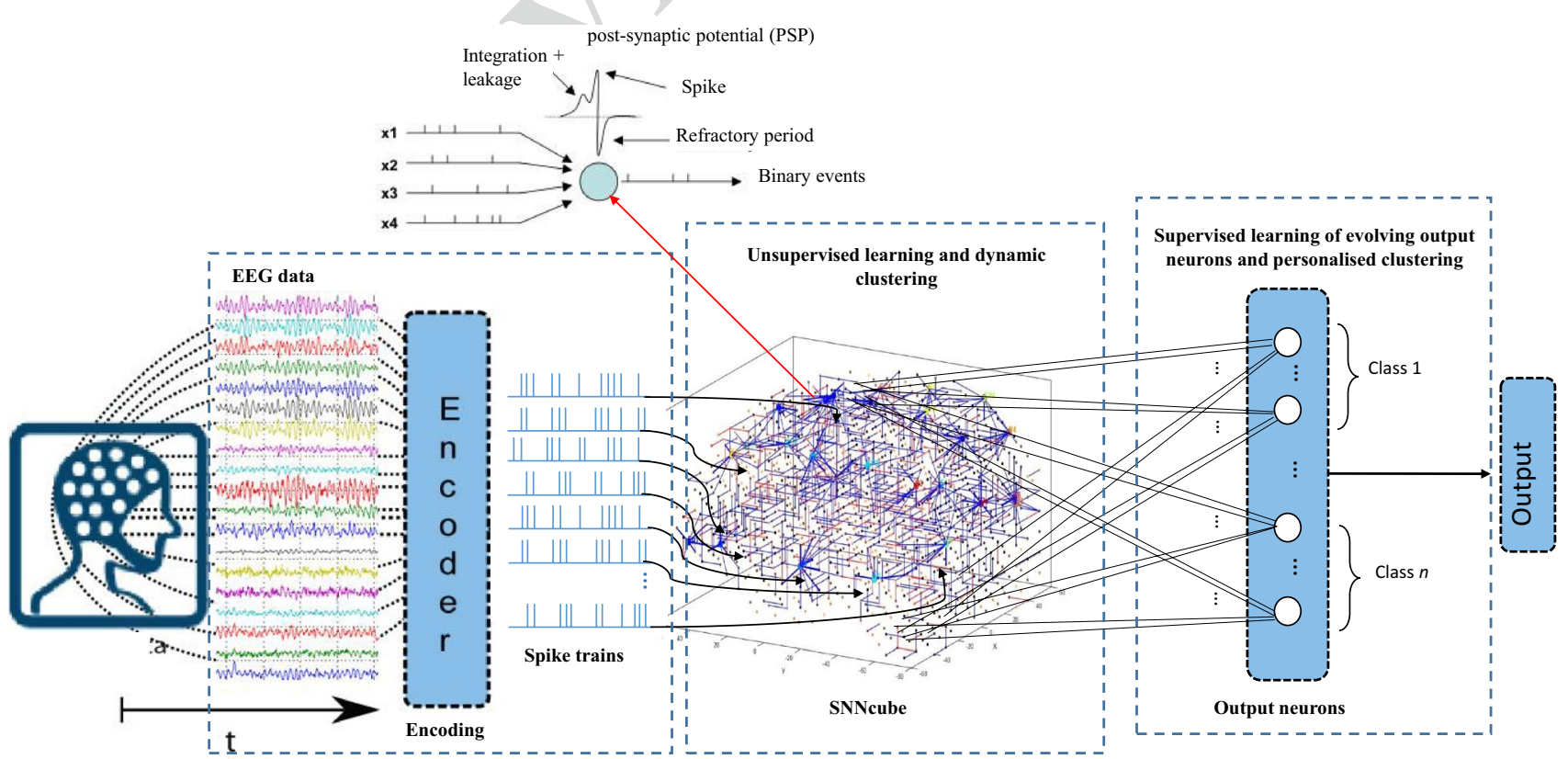

Fig. 1 A schematic block diagram of the NeuCube Architecture illustrated on EEG brain streaming data

\begin{tabular}{|l|l|l|l|l|}
\hline Journal : Large 12530 & Article No : 9178 & Pages : 17 & MS Code : EVOS-D-16-00033 & Dispatch : 27-3-2017 \\
\hline
\end{tabular}


$w_{i, j}=\frac{r_{i, j}}{d_{i, j}} ; \quad r \in \mathbb{R}_{[-1,1]}$

\subsection{Unsupervised learning in the SNNcube}

After SNNcube initialisation, unsupervised learning is performed using Spike-Timing Dependent Plasticity (STDP) learning rule (Song et al. 2000) as one implementation. STDP is one of the most popular example of Hebbian learning (Hebb 1949), which depends on the relative timing of pre- and post-synaptic action potentials. STDP learning rule is defined using the following relation:

$F(\Delta t)=\left\{\begin{array}{cc}A_{+} \exp \left(\Delta t / \tau_{+}\right) & \text {if } \Delta t<0 \\ -A_{-} \exp \left(-\Delta t / \tau_{-}\right) & \text {if } \Delta t \geq 0\end{array}\right.$

where $F(\Delta t)$ defines the synaptic modification elicited from a single pair of pre- and post-synaptic spikes separated by a time $\Delta t$. The parameters $\mathrm{A}+$ and $\mathrm{A}-$ define the maximum quantities of synaptic modification, which transpire when $\approx 0$. The parameters $\tau_{+}$and determine the ranges of pre-topostsynaptic inter spike intervals over which the synaptic strengthening and weakening occur (Song et al. 2000),

STDP learning stage is performed through transferring spikes (in time) across spatially located synapses and modifying the synapses over time. In this learning, a neuron's Post Synaptic Potential (PSP) increases by every input spike at time $t$ to reach the firing threshold which can be also a dynamical threshold as proposed in (Schliebs and Fiasche 2012). Once the PSP exceeds this threshold, the neuron fires and sends a spike to the other neurons that are connected to it. In STDP learning, if neuron $i$ fires before neuron $j$, the connection weight from neuron $i$ to neuron $j$ will increase, otherwise it will decrease. As a result, STDP adjusts the connection weights between neurons based on the relative timing of a particular neuron's output and input spikes. STDP learning encodes the 'hidden' spatiotemporal relations between SSTD streams in the form of "neuronal connections" and "spiking activities" in the SNNcube model. This information is used to define spatiotemporal similarity in the proposed dynamic clustering method.

\subsection{Supervised learning of evolving output neurons for classification}

For data classification/regression, dynamic evolving SNNs (deSNNs) (Kasabov et al. 2013) is used to train an output classifier based on the association between class labels and training samples. For each training sample, an output neuron is created and connected to each neuron in the SNNcube (shown in Fig. 1). After the unsupervised learning stage in the SNNcube is finished, the same data is propagated again through the trained SNNcube sample by sample. The spatiotemporal pattern of activation of the trained SNNcube evoked by a particular sample is used as input data to train an output neuron to recognize this pattern. The initial connection weight between a neuron $i$ from the SNNcube and an output neuron $j$ is defined by using the Rank-Order (RO) learning rule (Thorpe and Gautrais 1998). Through this rule, the first arrived spike to the output neuron $j$ would have the highest value:

$W_{i, j}(t)=\sum \bmod ^{\operatorname{order}(i)}$

where $\bmod$ is a modulation factor and $\operatorname{order}(i)$ is the order of the coming spikes to the connection between neurons $i$ and $j$.

While the RO learning will set the initial values of the connection weights $W_{i, j}$, the STDP rule will adjust these connection weights based on further incoming spikes. The connection weight $W_{i, j}$ is further modified by a small drift parameter value, so that at a next time $t$ if a spike arrives from neuron $i$ to neuron $j$, the weight $W_{i, j}$ will increase by a positive drift parameter value and if not, it will decrease by the drift value. The potential of neuron $j$ at time $t$ is calculated as follow:

$\operatorname{PSP}(j, t)=\sum \bmod ^{\text {order }(i)} W_{i, j}$

The details of the deSNN learning are published in (Kasabov et al. 2013).

\section{The proposed methodology for clustering of SSTD in a NeuCube SNN model}

In contrast to traditional clustering techniques, which are based on either spatial or temporal components and corresponding similarity measures (Kohonen 1998; Kasabov and Song 2002; Deboeck and Kohonen 1998; Kasabov 2007), we propose here a new approach for dynamic data clustering with respect to spatiotemporal similarity measures including both spatial and temporal components from the data that is learned in a SNN model. The proposed methodology is performed in both unsupervised and supervised learning modes and consists of the following clustering methods:

1. Dynamic clustering in an unsupervised learning mode in a 3D SNN model: dynamic spatiotemporal clusters, represented as either similar neuronal connections or similar temporal interactions between the spiking neurons in a SNN model during the unsupervised learning (Sect. 3.1).

2. Dynamic clustering in supervised learning mode related to individual outputs ("personalised"): (a) clusters of connections between the 3D SNN model 
from above and each individual output neuron, representing one class or one prototype from a given class; (b) clusters of spike time activity in the 3D SNN model from above related to each individual output neuron activation (Sect. 3.2).

These clustering phases are followed by analysis and interpretation of the created clusters for the discovery of new information about the SSTD and the processes that generated it.

\subsection{Dynamic clustering based on spiking activity and neuronal connectivity during unsupervised learning}

In order to apply the dynamic clustering of spatiotemporal data in a SNNcube, the cluster centres are predefined by the spatial locations of the data sources used as input variables (e.g. EEG channels in the case study presented later). During the unsupervised STDP learning in the SNNcube, spikes are transmitted between neurons across synapses that cause modifications of the connection weights. The more spikes are transmitted between two neurons $i$ and $j,\left(S_{i j}\right)$, the stronger the connection $\left(W_{i j}\right)$ becomes between them. During the clustering procedure, each neuron belongs to a cluster centre (which is the input neuron, corresponding to an input variable) that has the strongest connection or the most spikes received from this input neuron when compared with other input neurons.

Such created clusters are three dimensional and have different shapes as illustrated in Fig. 2a. The size and the shape of a cluster represent the importance of the cluster centre in the trained SNNcube model and thereforethe importance of the corresponding input variable in the SSTD.

In the SNNcube, the input neurons are allocated to the cluster centres and labelled by the input variables (e.g. EEG channels). The rest of the neuros in the SNNcube are unlabelled. The goal is to assign the cluster labels of the unlabelled neurons in the SNNcube. This procedure is addressed through the following steps:

1. An adjacency graph $G(V, E)$ is defined on the SNNcube, where vertex set $V$ represents the spiking neurons of the SNNcube and edges $E$ are weighted by either $S_{i j}$ or $W_{i j}$ when the clustering is based on the spiking activity or neuronal connectivity respectively. Note that stronger $E_{i j}$ represents stronger intraction and more information shared between neurons $i$ and $j$ in SNNcube.

2. Given graph $G$ and starting vertex $v_{s}$, a random walk (Tu et al. 2014) in $G$ of length $n$ is defined as a randomised process in which, starting from the vertex $v_{s}$, we repeat $n$ times a step that consists of choosing a random neighbour of the vertex $v_{s}$. We define $p_{v}^{n}$ to be the probability that we chose vertex $v$ after $n$ steps of random walk. If random walk starts from vertex $v_{s}$, which represents one input neuron in the SNNcube, the initial distribution of the random walk can be defined as:

$p_{v}^{0}=\left\{\begin{array}{cc}1, & \text { if } v=v_{s} \\ 0, & \text { otherwise }\end{array}\right.$

At a further step $n$ of this random walk, the probability distribution is defined as:

$\forall v \in V: P_{v}^{n+1}=\sum_{(u, v) \in E} \frac{E_{u v}}{d(u)} P_{u}^{n}$

where $d(u)$ is the weighted degree of vertex $u$, that is, the sum of weights of adjacent edges.

3. The evolution of the probability $P_{v}^{n}$ continues as a function of $n$ until it converges, i.e. $P_{v}^{n+1}=P_{v}^{n}$. For every vertex $v \in V$, the obtained $p_{v}$ represents the membership to the input vertex. At the end, each vertex $v \in V$ will belong to a starting vertex (input neuron) from
Fig. 2 a Neuronal clusters created through unsupervised clustering in a SNNcube; b measuring spike exchanges between neuronal clusters (the thicker the lines, the more spikes are exchanged between the clusters). The example uses results from the case study simulation of brain spatiotemporal data measured as EEG through 26 channels

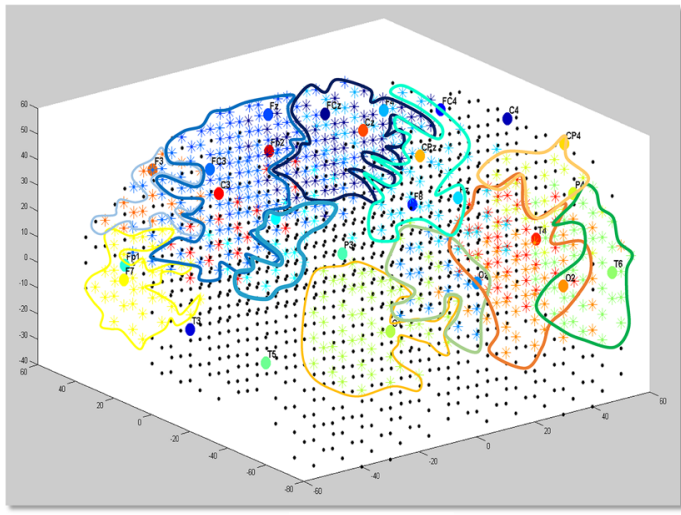

(a)

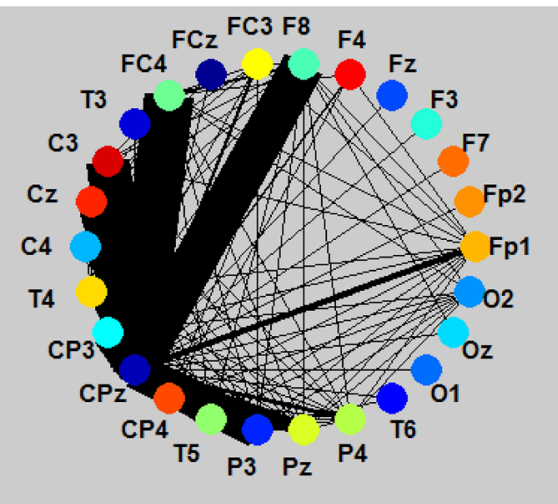

(b) 
which it obtained the highest membership $p_{v}$. This algorithm is mathematically implemented in (Tu et al. 2016; Zhou et al. 2004).

In this paper, the above technique is applied at each time unit $t$ of the STDP learning process to generate dynamic clusters which are formed with respect to $S_{i j}$ or $W_{i j}$ of the SNNcube. This clustering also allows to represent the level of interaction between clusters (corresponding to brain areas in the example of Fig. 2a) measured as number of spikes exchanged between the clusters-see Fig. $2 b$. In the graph presented in Fig. $2 b$, the thicker the connected line between two variables is, the more the spikes exchanged.

\subsection{Dynamic clustering of the SNNcube connectivity and temporal activity based on supervised learning of individual evolving outputs ("Personalised")}

As discussed in Sect. 2, after the unsupervised learning in the SNNcube is finished, the same data is propagated again through the trained SNNcube sample by sample for supervised learning. For each training sample, we create an output neuron and connect it to each neuron in the SNNcube. An output neuron is associated with a single input spatiotemporal sample or prototypes of samples from a given class. As a partial case it can represent brain or other data from one person. Initial connection weights of the neurons from the SNNcube to an output neuron $j$ are set according to RO rule in which the earlier spikes arriving to a neuron $j$ resulting in a higher initial connection weight. The output neuron is trained to recognize the spatiotemporal pattern of activity in the already trained SNNcube that is triggered when an input pattern corresponding to this individual is propagated through the SNNcube.

Through the clustering, neurons in the SNNcube are labelled by their connection weights to the output neuron $j$.

The stronger the connection from a neuron $i$ from the SNNcube to an output neuron $j$, the higher the impact of the spiking activity of neuron $i$ is on the activation of the output neuron $j$ representing the SSTD related to brain activity of an individual subject $j$. In order to define the cluster centres in SNNcube, we can choose a number of neurons with different label values (between the minimum and the maximum connection weights). This clustering is performed through grouping the neurons in the SNNcube into clusters of similar connection weights to neuron $j$. In this way the importance of the brain areas can be ranked according to the performance of an individual $j$ during a cognitive task.

During the supervised learning, when an input data sample related to the performance of an individual $j$ is entered into the already trained SNNcube, the order in which the neurons in the SNNcube spike reflects the temporal activities captured in the SSTD of the individual $j$. Capturing this order in groups of neurons that spike at a similar time period results in clusters of neurons in the SNNcube. In this procedure, the neurons of SNNcube will be ranked by the temporal order in which they spike. The minimum neuron rank is 1 and the maximum rank is when the last SSTD time point comes into the SNNcube for supervised learning. We selected a number of neurons from the ranked SNNcube as cluster centres to capture groups of neurons with similar spiking orders to the centres. The cluster members are selected if they have close proximity (more than a similarity threshold) to the cluster centre. These clusters can be used to understand the timing of the sequential brain activities when a particular individual $j$ is performing a cognitive task. Sect. 3.2 is illustrated in Sect. 6 on three individuals, from the case study data, belonging to three classes. Figure 3 shows a block diagram of the proposed clustering methods.

\section{Feasibility study of the proposed clustering methods on a case study EEG data}

In order to validate the proposed methods, we demonstrate it on a case study of EEG data which was recorded during a cognitive GO-NOGO task performed by three groups of subjects: healthy subjects $(\mathrm{H})$, opiate user subjects $(\mathrm{OP})$, and opiate users under methadone maintenance treatment (M). Prior to commencing this research, ethical approval was granted by the "Northern X Regional Ethics Committee of New Zealand" and informed consent was given by all participants. Identifying information of participants including names, initials, etc. are not reported in the paper.

A cognitive GO-NOGO task has been used, in which participants were repeatedly presented with the word 'PRESS' (for $500 \mathrm{~ms}$ ). The colour of the word 'PRESS' was presented randomly in either red or green. Participants were instructed to respond by pressing a button in response to the word that appeared in green $(\mathrm{GO})$ and not respond to the word that appeared in red (NOGO). The EEG data was recorded via 26 EEG channels: Fp1, Fp2, Fz, F3, F4, F7, $\mathrm{F} 8, \mathrm{Cz}, \mathrm{C} 3, \mathrm{C} 4, \mathrm{CP} 3, \mathrm{CPz}, \mathrm{CP} 4, \mathrm{FC} 3, \mathrm{FCz}, \mathrm{FC} 4, \mathrm{~T} 3, \mathrm{~T} 4$, T5, T6, Pz, P3, P4, O1, O2, and Oz.

The GO-NOGO task was displayed and simultaneously the event-related potentials (ERPs) were recorded. We extracted 75 EEG time points per subject, each corresponds to grand mean average. In this case the EEG data analysis is in time domain. Based on the most literature about GO/NOGO task, the brain response inhibition, as a core executive function, is expected to be observed in prefrontal, frontal, dorsal, ventral, and parietal regions, which are related to human response inhibition. On the other hand, psychological reports showed there is a direct

\begin{tabular}{|l|l|l|l|l|}
\hline Journal : Large 12530 & Article No : 9178 & Pages : 17 & MS Code : EVOS-D-16-00033 & Dispatch : 27-3-2017 \\
\hline
\end{tabular}




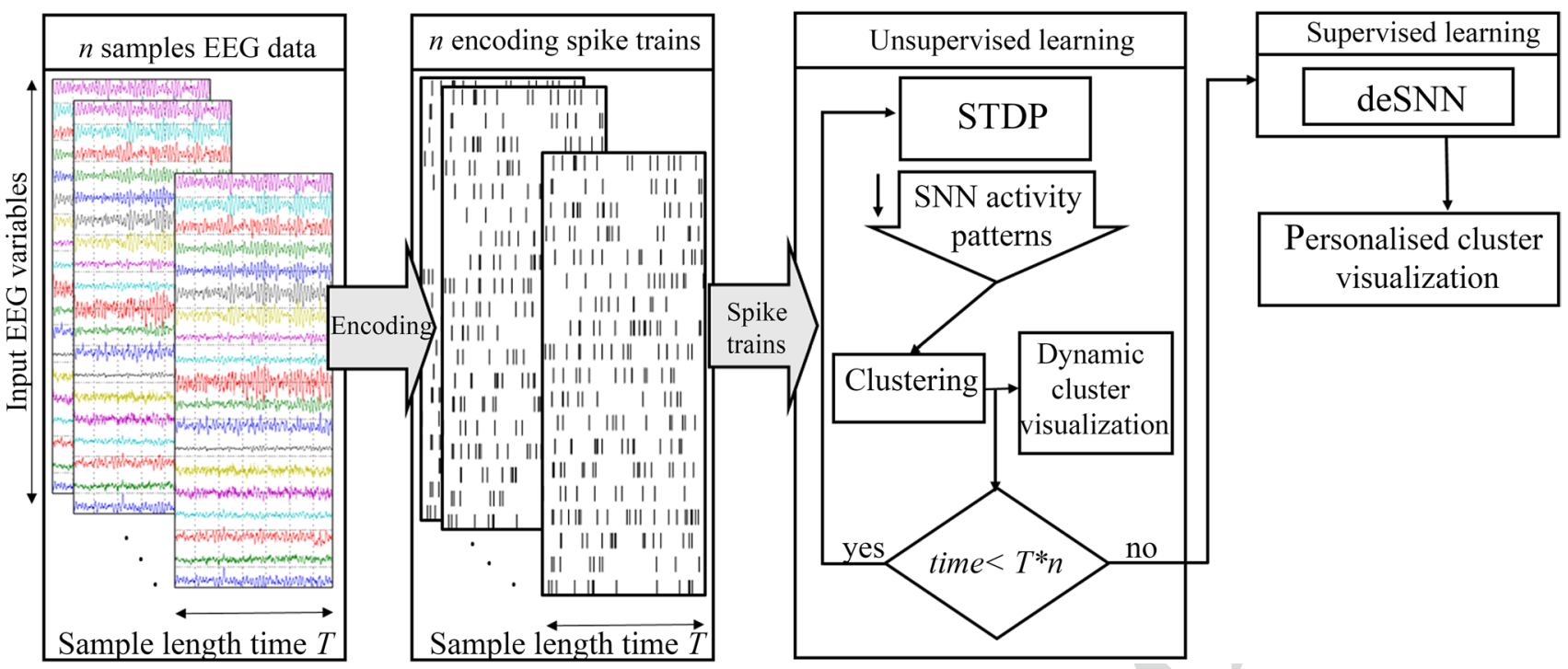

Fig. 3 A block diagram of the clustering methods in the NeuCube SNN architecture

relation between the response reduction in prefrontal cortex magnitude and addictive behaviour, due to the drug effects on these particular brain functions.

In this study, we aim to apply the proposed clustering methods to the EEG case study, to illustrate the differences between the clusters in terms of the cluster evolution pattern, cluster creation time, and cluster size across the subject groups when performed GO versus NOGO trials.

We performed six EEG sample files, each containing EEG data captured from one group $(\mathrm{M} / \mathrm{OP} / \mathrm{H})$ per cognitive task (GO versus NOGO). Each sample file was entered separately into the SNNcube for unsupervised training. Every learning was started by entering the first EEG time point to train the SNNcube and it was finished after entering the final EEG time point. Simultaneously, neuronal clusters were created with respect to the neuron spiking activity and connectivity in the SNNcube.

\section{Application of the proposed dynamic clustering in the unsupervised learning mode of the SNNcube on EEG data for the case study problem}

\subsection{Dynamic clustering of EEG data in a SNNcube model for the case study problem}

The SNNcube clusters were formed and updated with every new input EEG time point entered, frame by frame. This process can be traced and analysed in terms of:
1. The order in which input EEG channels formed the clusters, related to the order of activity of the corresponding areas of the brain.

2. The evolution of the size of the clusters, related to the importance of the activity of brain areas over time.

Figure 4 shows step-wise visualisation of the neuronal cluster evolution corresponding to the 26 EEG channels in the SNNcube models. Figure 4a shows how the input neurons of the SNNcube are allocated to the respective EEG channels for transferring the spike trains. It also shows the evolution of the clusters for four selected time points during the unsupervised learning of the SNNcube with EEG data of $\mathrm{H}$ group when performing a GO task. Cluster creation started from predefined centroids and clusters were changed after every input EEG time point is entered into the SNNcube. Since there were 21 healthy subjects, and 75 EEG time points were captured from every subject, the last time point of the training data was $21 \times 75=1575$.

Figure $4 b$, c represent the dynamic clustering of the EEG data related to $\mathrm{M}$ and OP subjects in GO trials with a total number of $29 \times 75=2175$ and $18 \times 75=1350$ EEG time points respectively. The reason that we have chosen different time frames in our visualisation is the time differences in clusters creation across the subject groups with respect to their EEG data. Once new clusters were created during the NeuCube training, a new figure was captured to display the step-wise changes in the cluster evolution. These results show that when a SNNcube is training with EEG data of $\mathrm{H}$ group in GO task, the first created clusters correspond to $\mathrm{Fz}$ and $\mathrm{FCz}$ channels after entering the 8th EEG time point to the learning process. Those neurons that are clustered

\begin{tabular}{|l|l|l|l|l|}
\hline Journal : Large 12530 & Article No : 9178 & Pages : 17 & MS Code : EVOS-D-16-00033 & Dispatch : 27-3-2017 \\
\hline
\end{tabular}



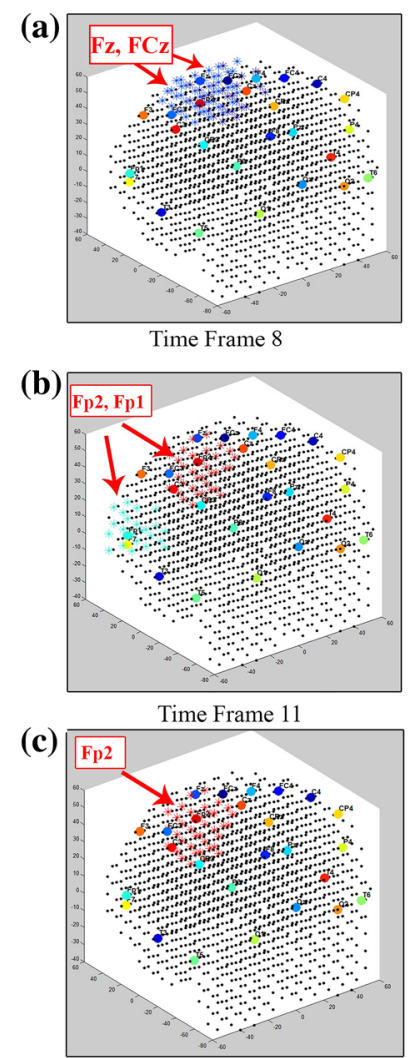

$$
\text { (d) }
$$

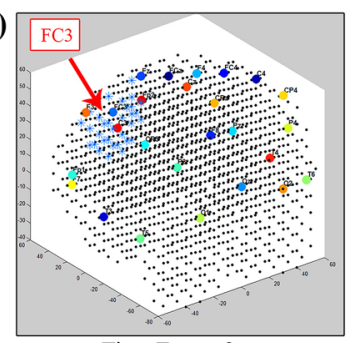

(e)
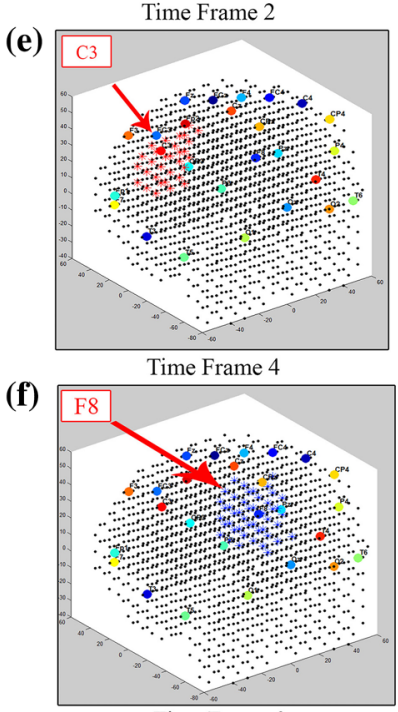

Time Frame 9

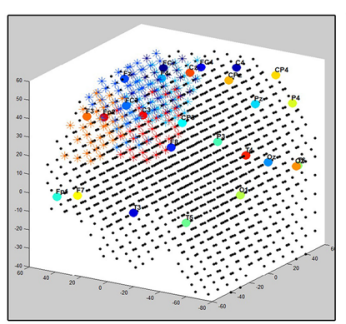

Time Frame 9
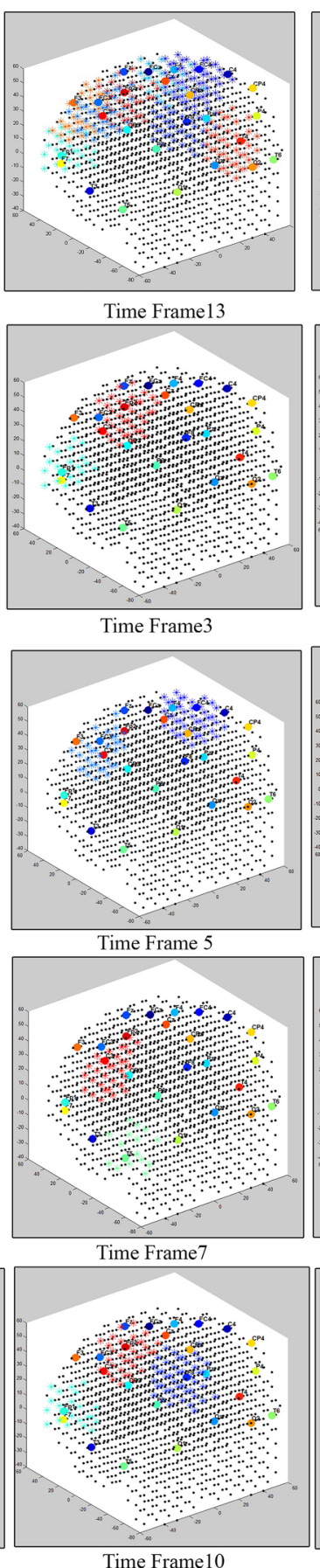
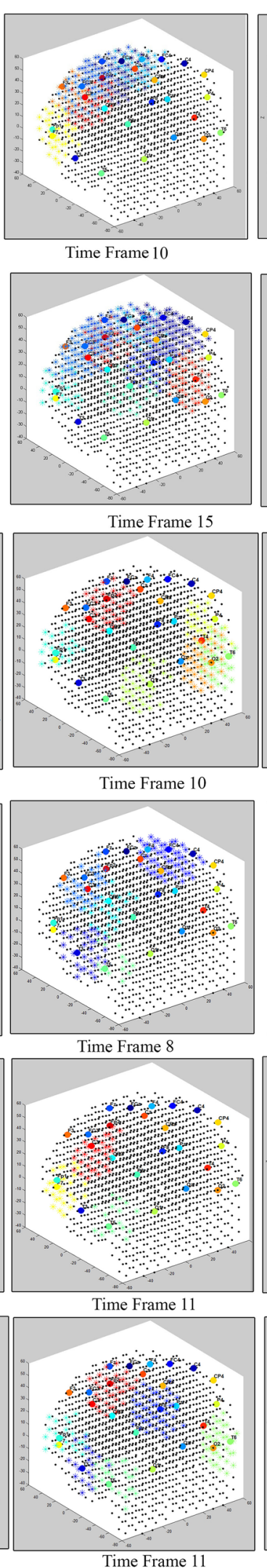

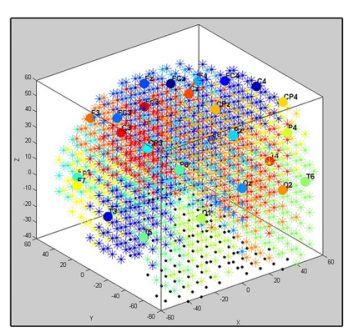

Time Frame 1575

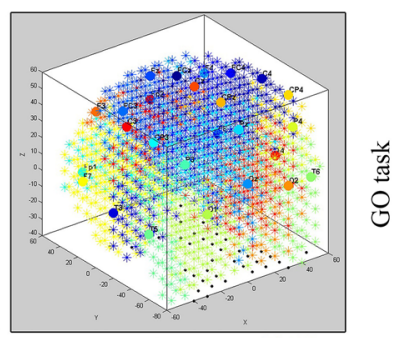

紊
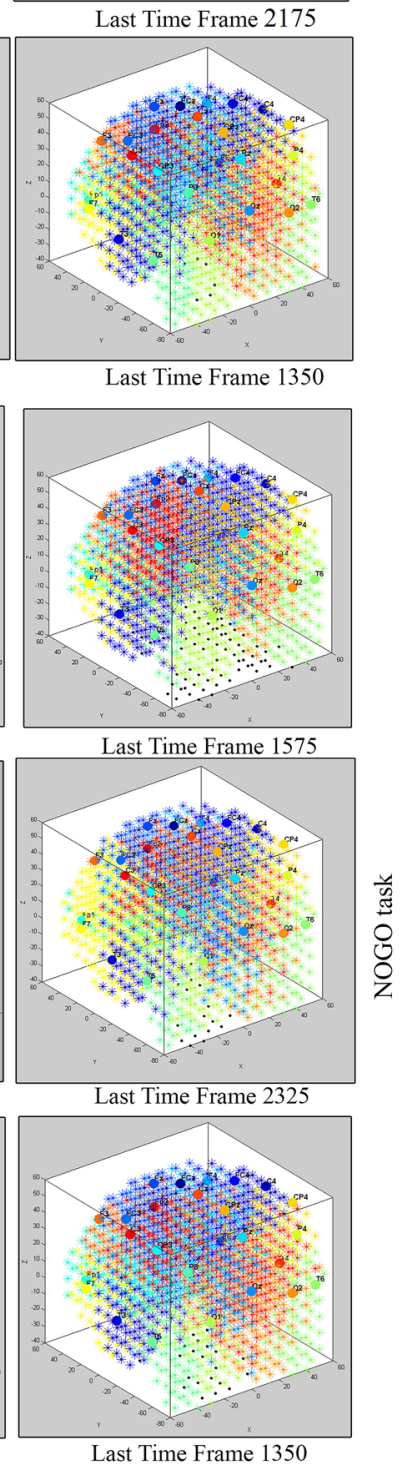

Fig. 4 a-c The cluster evolution during the unsupervised learning with EEG data of H, M and OP subjects in the GO task, $\mathbf{d}-\mathbf{f}$ the neuronal cluster evolution of $\mathrm{H}, \mathrm{M}$ and OP subjects in the NOGO task 
by $\mathrm{Fz}$ and $\mathrm{FCz}$ channels have received the most number of spikes from these corresponding channels by this moment of the learning process. On the other hand, for M group, the first neuronal clusters are created by FP1 and FP2 channels at 11th time points. In case of OP subjects, the first cluster is created by FP2 at the 2nd EEG time point.

Figure $4 \mathrm{~d}-\mathrm{f}$ are related to the NOGO trials. For H group, there are a total number of $21 \times 75=1575$ EEG time points and the first created cluster is related to the FC3 channel at 2nd EEG time point. It represents that more spikes were transferred into the SNNcube via FC3 channel at this time. Therefore, those neurons located around this channel are clustered faster than the other neurons in the SNNcube. As the frontal areas of the brain are involved in inhibition functions, our finding may indicate that $\mathrm{H}$ group has successful inhibition with less response time in performing the NOGO trials. However, the first clusters for $\mathrm{M}$ and $\mathrm{OP}$ groups were generated later than the clusters in $\mathrm{H}$ group and they belonged to the $\mathrm{C} 3$ and F8 at 4th and 9th EEG time points respectively. These results show that in $\mathrm{M}$ and $\mathrm{OP}$ groups, slower response was observed from frontal regions and consequently less number of spikes were entered into the SNNcube when compare it with $\mathrm{H}$ group. There were $31 \times 75=2325$ and $18 \times 75=1350$ EEG time points for $M$ and OP group respectively.

The neuronal clusters evolved in the SNNcube during unsupervised learning can be also statistically compared in terms of the size (number of neurons that belong to each cluster) and also in terms of the cluster creation time. The clusters were scaled up or down with respect to the number of neurons associated with every input EEG variable. A bigger cluster contains larger number of spiking neurons around the centre, which means more spikes transmitted via this centre to the SNNcube. By comparing the number of neurons that belong to each cluster centroid, we can differentiate the dynamic brain activity captured via different EEG channels across the subject groups in GO versus NOGO trials. Figure 5a visualises how the cluster size of two EEG variables change during the unsupervised learning in SNNcube. The horizontal axis represents the number of EEG data points entered to the SNNcube training via input neurons corresponding to the EEG channels. The vertical axis represents the number of neurons that belong to each cluster at each time unit of the SNNcube learning process.

As observed in prefrontal electrodes (as the potentially best candidate to reflect inhibition-related cognitive activity), there is a specific reduction in the prefrontal and frontal activity in the $\mathrm{M}$ group in NOGO task (illustrated by red plot). Less number of neurons in F3 and FP2 clusters may represent that $\mathrm{M}$ group exhibited significant reduction of their attention within the frontal region. This clustering trend allows us to understand the differences between subject groups by distinguishing their brain activities as different clusters.

Using the proposed clustering methods, we can also extract important EEG variables that contribute to generating large clusters.

Figure 5a showed the dynamics of the cluster size changes during the presentation of the EEG data of a particular order of the subject data. Two questions may arise in this regards.

Would cluster evolution be different within a subject group?

Would the order of presentation of subject data influences the final clustering?

To address these questions, we performed the clustering experiment ten times for each subject group using random order of the subject data as shown in Fig. 6. It can be seen that different clusters based on EEG channels have different variability across subject groups and also within a subject group.

In the SNNcube, the neuron's Post Synaptic Potential (PSP) increases by every input spike arrived to the neuron at time $t$. Once the PSP reaches a threshold, neuron emits the output spike. The total spiking rate emitted by the neurons within a cluster as well as their total PSP rate are reported in Fig. 5b for one randomly selected cluster (FP1 channel). This result allows us to study the pattern of the cluster creation more in details by looking at the number of spikes produced within a cluster and also the pattern of the neurons' PSP during the learning.

\subsection{Analysis of dynamic brain processes through mapping neuronal clusters from the NeuCube model back to brain areas}

Using the NeuCube models, we can map the obtained neuronal clusters into the corresponding brain areas for a better localization of the brain activity sources and to precisely show which areas of the brain are associated to each cluster. In order to interpret the dynamic clusters in terms of associated brain areas, we have used the generic Talairach brain template (Koessler et al. 2009; Lancaster et al. 2000; Talairach and Tournoux 1988). In Fig. 7, a trained SNNcube is labelled by eight Talairach brain areas, namely: frontal lobe in yellow-green; temporal lobe in pink; parietal lobe in light-blue; occipital lobe in red; posterior lobe in light yellow; sublobar region in orange; limbic lobe in green; and anterior lobe in blue.

Figure 7a illustrates the connected neurons in two randomly selected clusters (FC3 and FC4) in healthy versus OP groups in GO task. The stronger the connections created between neurons, the more spikes transmitted between them. Figure $7 \mathrm{~b}$ shows the strongest connections (larger than a threshold) within the clusters. Less connections 


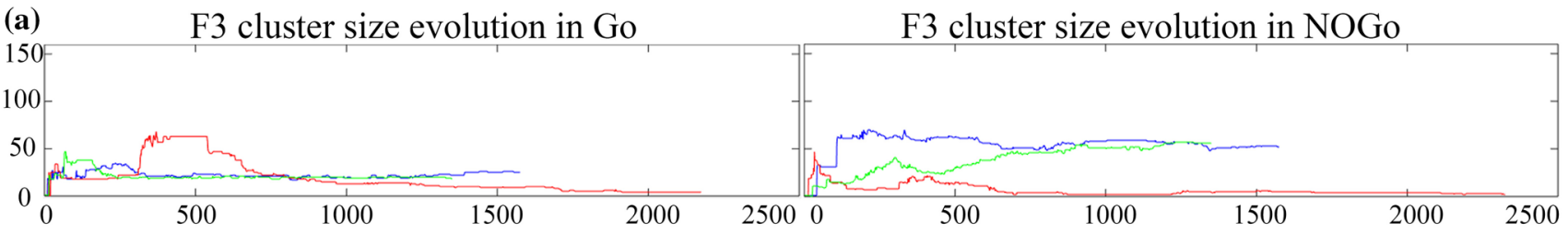

FP2 cluster size evolution in Go

FP2 cluster size evolution in NOGo

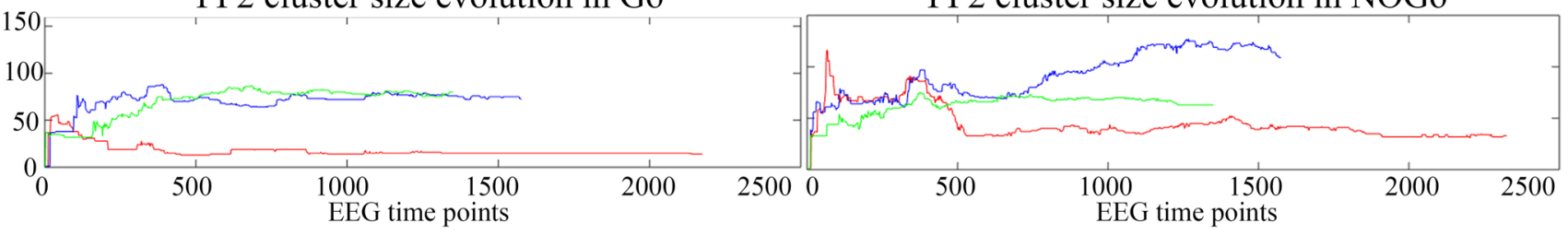

(b) H: Total spike rate of FP1 MMT: Total spike rate of FP1 OP: Total spike rate of FP1

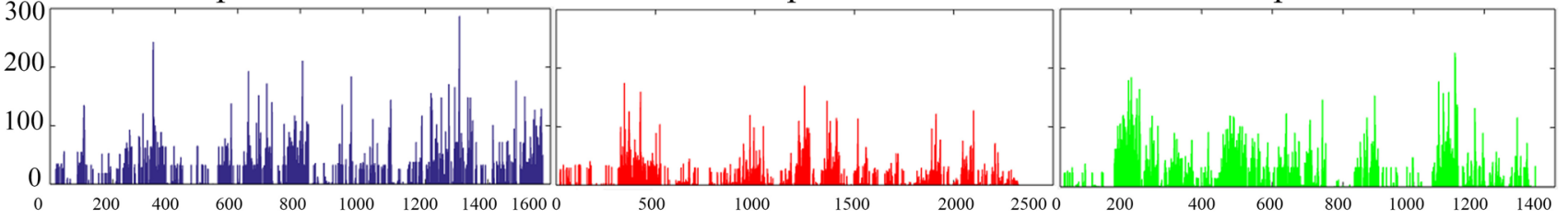

H: Total PSP rate of FP1

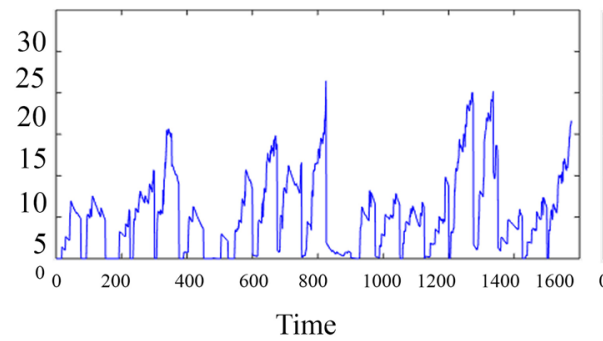

MMT: Total PSP rate of FP1

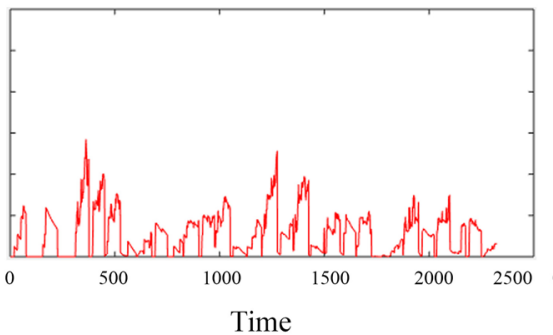

OP: Total PSP rate of FP1

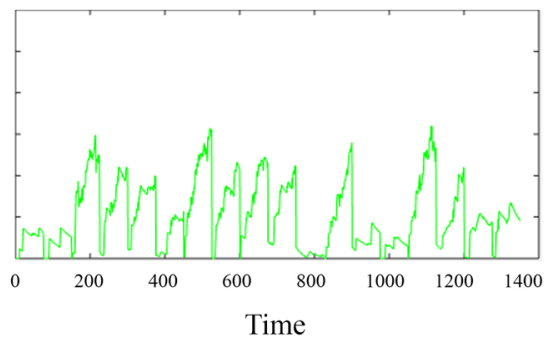

Fig. 5 a The size of the clusters, centred at the input neurons corresponding to EEG channels (F3, FP2), changes during the cluster evolution in the SNNcube while training on EEG data of $\mathrm{H}$ (blue), M (red), OP (green) subjects. b Total spike rate and PSP rate of the neurons within the FP1 cluster during the unsupervised learning of EEG data in NOGO task. Blue $\mathrm{H}$, red $\mathrm{M}$, and green OP. The LeakyIntegrate and Fire (LIF) patterns of spiking neurons can be captured from both spiking rate and PSP rate captured for OP group represents less functional brain activities observed when OP group was expected to response in the GO task in comparison with healthy group. As it is seen from Fig. 7b, c, greater connections were detected in the neuronal clusters of the healthy group, significantly in the frontal areas. Our findings represent that healthy group was able to perform more accurate responses in the GO task. Figure 7d shows the static information of dynamic cluster creation (size and time) in one particular order of subject data. It allows us to trace the sequence of the functional brain activities associated with different brain areas. In this figure we can see when the healthy group deals with GO task, the clustering of the neurons starts from the functional brain activities generated in middle frontal lobe (37 and 26 neurons of SNNcube belong to the $\mathrm{Fz}$ and $\mathrm{FCz}$ clusters respectively at 8 th time point of learning process) and then followed by the inferior and the superior frontal gyrus $(23,35,43,27,26,29$ neurons belong to $\mathrm{F} 3, \mathrm{Fz}, \mathrm{F} 4, \mathrm{Fc} 3, \mathrm{Fcz}$ and $\mathrm{C} 3$ clusters respectively at 9 th time point).

\section{Dynamic clustering of SNNcube patterns in a supervised mode for individual output neurons of a NeuCube model on the case study problem}

As described in Sect. 2.3, the second training stage is to train the output classifier using class label information associated with the training samples. The deSNN is used as output classifier is illustrated in Fig. 8 that shows for every training sample representing EEG data of one subject, one output neuron is evolved and labelled by its class label (red: healthy subjects; green: M subjects; and

\begin{tabular}{|l|l|l|l|l|}
\hline Journal : Large 12530 & Article No : 9178 & Pages : 17 & MS Code : EVOS-D-16-00033 & Dispatch : 27-3-2017 \\
\hline
\end{tabular}


Number of neurons that belong to each cluster in $\mathrm{H}$ group

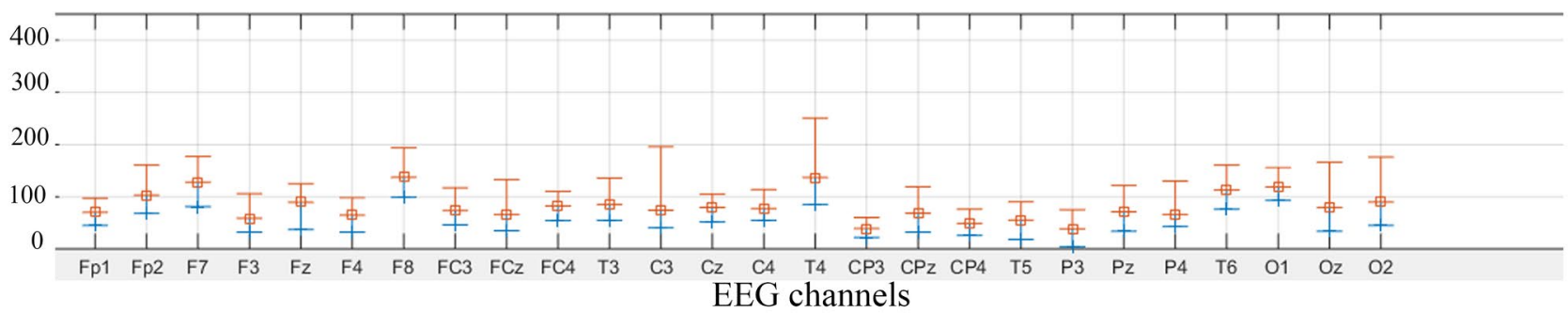

Number of neurons that belong to each cluster in MMT group

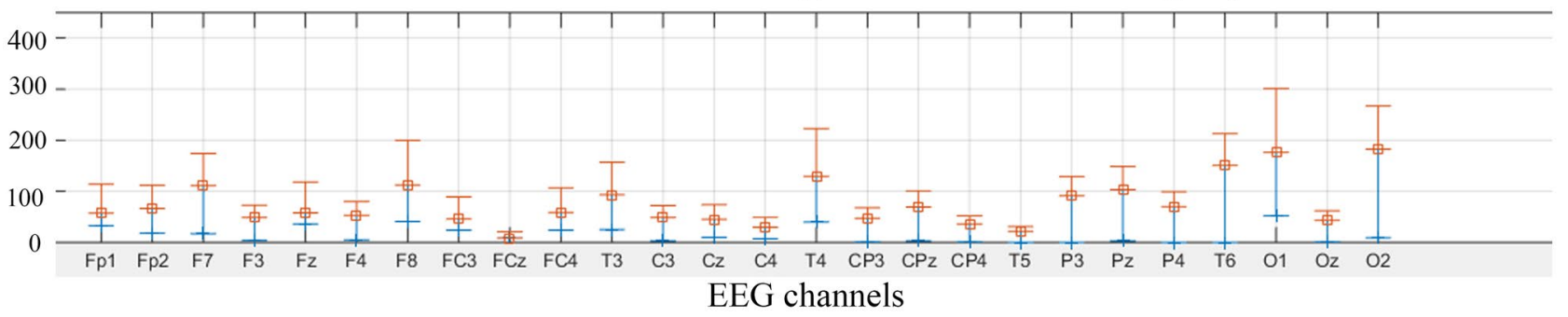

Number of neurons that belong to each cluster in OP group
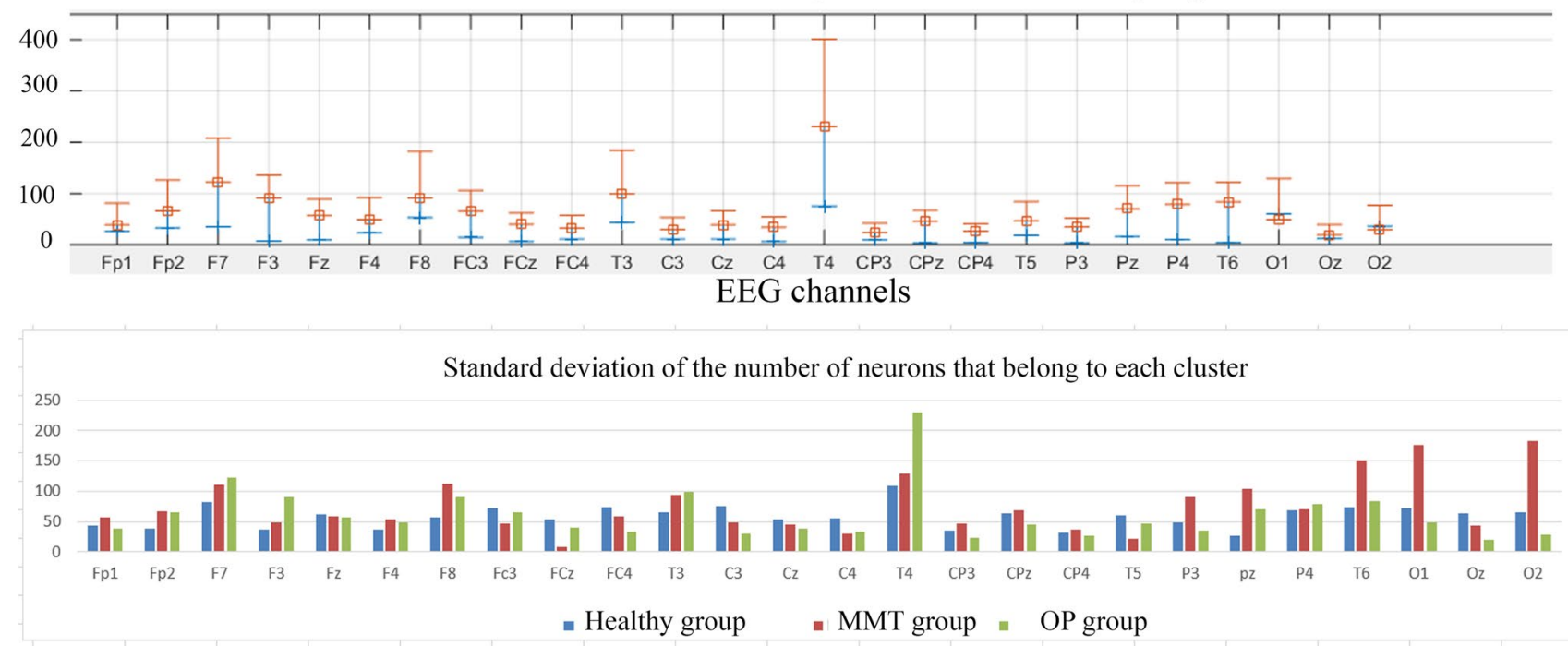

Fig. 6 Minimum, maximum and mean of the number of neurons that belong to each cluster. The dynamic clustering is run 10 times for each group using random order of the subject data presentations.

blue: OP subjects). The output neurons are connected to all neurons of the SNNcube and the connection weights between them are initialised based on the RO rule. This learning rule endows a higher priority to the first spike coming to the output neuron. After the first spikes entered, the connection weights are modified according to the further incoming spikes to the output neurons. When the supervise learning is completed, the connection weights between SNNcube and the output neurons are stablished. These connection weights are visualised by coloured neurons. Brighter neurons mean larger
The standard deviation $\sigma$ is reported in the last row. Higher $\sigma$ mostly observed in $\mathrm{M}$ and OP groups

connection between neuron $i$ from SNNcube to the output neuron $j$.

In order to represent an individual performance, we clustered the connections weights between the SNNcube's neurons and an output neuron $j$ based on two aspects:

a) Clusters reveal the importance of the areas of neurons in the SNNcube for the activation of this output neuron.

b) Clusters reveal the order of activity of neurons in the SNNcube according to their time of spiking.

\begin{tabular}{|l|l|l|l|l|}
\hline Journal : Large 12530 & Article No : 9178 & Pages : 17 & MS Code : EVOS-D-16-00033 & Dispatch : 27-3-2017 \\
\hline
\end{tabular}


Figure 8 shows for an individual subject (selected output neuron $j$ ), each neuron $i$ from the SNNcube is labelled by the connection weight value between $i$ and the output neuron $j$. In the labelled SNNcube, a number of neurons with different label values (selected between the minimum and the maximum connection weights) are selected as the cluster centres. Those neurons that have similar label value to the centre (less than a distance threshold) are selected as the cluster members.

In this example, for one individual healthy subject in NOGO task, the biggest cluster is located in the frontal areas which are associated with brain cognitive inhibition. The personalised clusters represent the importance of the brain areas corresponding to this particular subject's performance during the cognitive NOGO task. This finding is consistent with the literature that cognitive inhibition processes are ascribed to the frontal and prefrontal cortex that is fundamental for healthy neuropsychological functions. However, this observation is different for the selected $\mathrm{M}$ and OP individuals.

Figure $8 \mathrm{~b}$ shows that the SNNcube is labelled by the temporal order in which the neurons spike.

For one individual healthy subject in NOGO task, we selected 5 neurons with different label values between 1 and 75 (there are 75 EEG time points per subject). In this case, we can see different clusters are captured based on the similarity in the neuron temporal spiking time. The brighter neurons mean they spike earlier. Cluster members are those neurons with close distance (less than a distance threshold) to the centres. The clusters can be interpreted to discover which areas of the brain were activated earlier. For example, for the selected healthy subject, we can see those neurons located in the visual cortex are brighter, which means they spiked earlier. These cognitive processes were then followed by spikes at neurons located in central parietal and superior frontal areas. Our findings in Fig. 8b prove that the NeuCube clustering is supported by the neuroscience literature that reported visual perception initiates as soon as the eye transfers light to the retina, where it is observed by a layer of photoreceptor cells (Carter 2014). Compared to the M and OP individuals, we conclude that the visual cortex is activated first, but not many early spikes were captured in the frontal areas. This result may suggest the cognitive inhibition impairment in those subjects with the history of drug usage.

\section{Comparative analysis of the proposed dynamic, evolving clustering methods with SOM}

Here we demonstrate the principle differences between the proposed clustering methods and the method of clustering in Self-Organizing Maps (SOM) (Kohonen 1998;
Deboeck and Kohonen 1998)_one of the most popular method in computational intelligence. SOM are topological maps trained on a sequence of vector based data. Here we have applied SOM [available at (Hassinen 2015)] to the case study used in the paper of EEG data of healthy, $\mathrm{M}$ and OP subjects in GO task. In the healthy group, there are 21 subject data samples, each contains 26 EEG channels measured during 75 time points. We reorganized the EEG data of every subject as a vector of 130 elements. For each EEG channel, the 75 time points are aggregated into 5 time points. Therefore, 26 channel $\times 5$ time points $=130$ elements involved for each sample. The training data used for SOM has 21 vectors representing 21 healthy subjects, each vector of 130 elements (Fig. 9). The neurons in the trained SOM are clustered into nine clusters. These clusters represent which input vectors are similar to each other, but it does not reveal any spatiotemporal information related to the brain processes. A single individual sample will be mapped as a point on the SOM that does not reveal any dynamic information related to the performance of this individual. This is a significant contrast to the proposed in this paper clustering methods.

\section{Discussions and future work}

In this study, a generic SNN methodology was proposed as a novel approach to dynamic clustering of SSTD. The methods are based on the following scheme:

Dynamic processes (e.g. brain processes; seismic activities, etc.) $\rightarrow$ Spatio/Spectro Temporal Data $($ SSTD) $\rightarrow 3 D$ NeuCube model creation $\rightarrow$ NeuCube model clustering $\rightarrow$ Analysis of the data and the processes that generated it $\rightarrow$ Cluster update on new data.

The paper introduces two new methods for: (1) dynamic clustering in an unsupervised learning mode in 3D SNN model; (2) dynamic clustering in a supervised learning mode for individual outputs ("personalised").

Dynamic clusters represented similar spatiotemporal changes (events) in a SNN model during unsupervised learning. This method captures significant information about SSTD as it records the exact time in which a cluster was formed and it reveals how this cluster's shape changes over time. The cluster size and the time of creation represent the importance of the input variables (e.g. EEG channels) at different time $t$ of the learning process, providing insights into the input data structures and processes. This clustering also was used to investigate the dynamic patterns of spiking activity rate and membrane potential inside of each cluster over time.

The proposed supervised learning clustering method for individual output neurons (representing class or prototype labels) reveals two aspects of the data: (1) the importance
859 
(a)

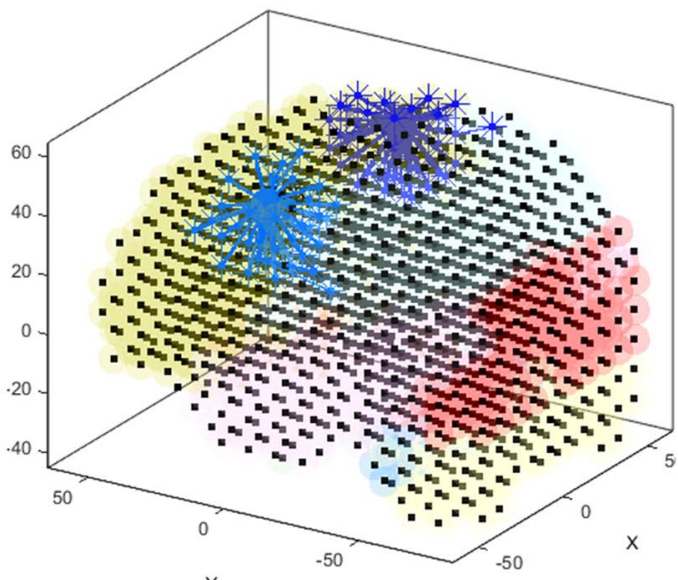

Healthy Subjects in GO

(b)

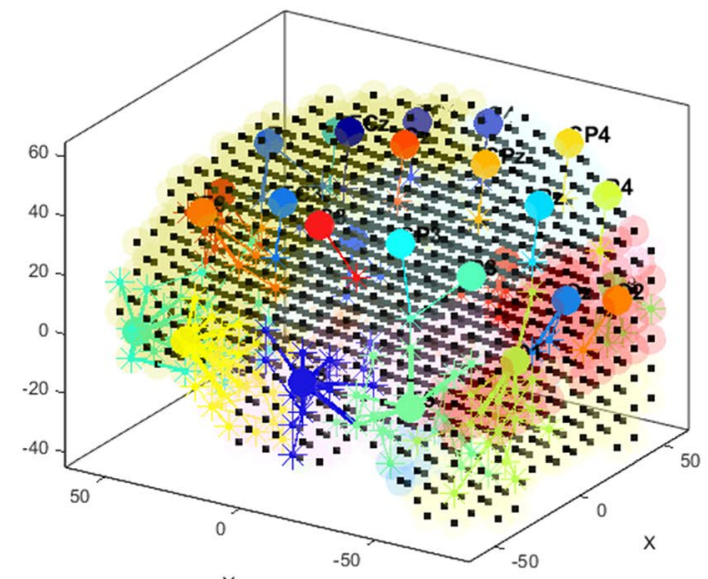

Healthy Subjects in GO

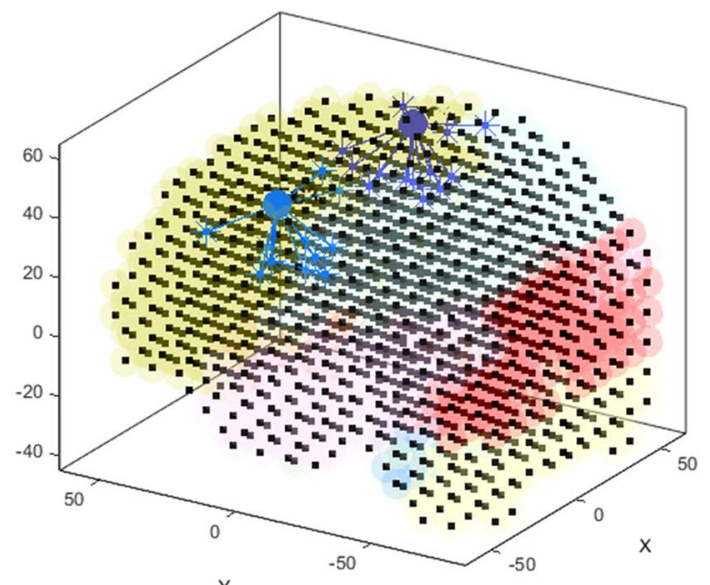

OP Subjects in GO

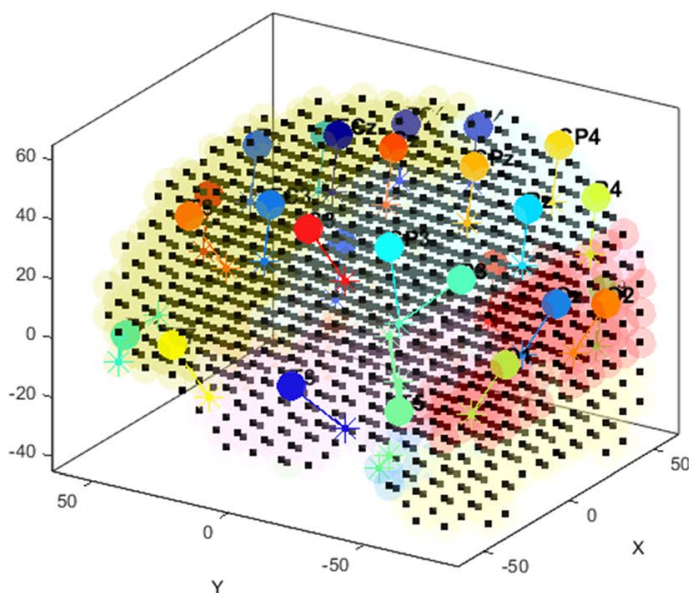

OP Subjects in GO

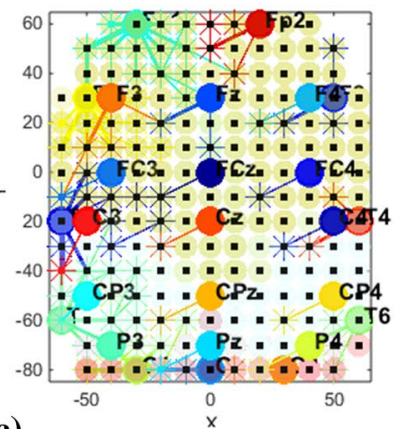

(c)

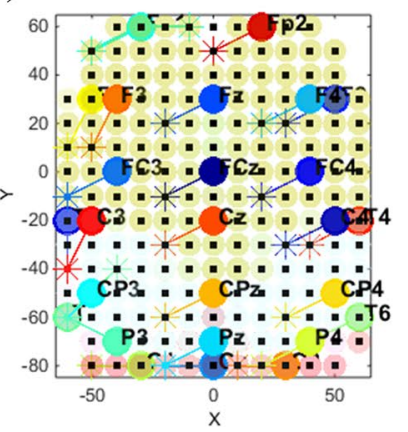

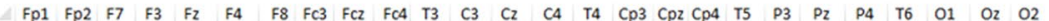
$\begin{array}{llllllllllllllllllllllllllllll}1 & 1 & 1 & 1 & 1 & 1 & 1 & 1 & 1 & 1 & 1 & 1 & 1 & 1 & 1 & 1 & 1 & 1 & 1 & 1 & 1 & 1 & 1 & 1 & 1 & 1 \\ 2 & 1 & 1 & 1 & 1 & 1 & 1 & 1 & 1 & 1 & 1 & 1 & 1 & 1 & 1 & 1 & 1 & 1 & 1 & 1 & 1 & 1 & 1 & 1 & 1 & 1 & 1\end{array}$ \begin{tabular}{l|l|l|l|l|l|l|l|l|l|l|l|l|l|l|l|l|l|l|l|l|l|l|l|l|l|l|l}
2 & 1 & 1 & 1 & 1 & 1 & 1 & 1 & 1 & 1 & 1 & 1 & 1 & 1 & 1 & 1 & 1 & 1 & 1 & 1 & 1 & 1 & 1 & 1 & 1 & 1 & 1 \\
3 & 1 & 1 & 1 & 1 & 1 & 1 & 1 & 1 & 1 & 1 & 1 & 1 & 1 & 1 & 1 & 1 & 1 & 1 & 1 & 1 & 1 & 1 & 1 & 1 & 1 & 1
\end{tabular} $\begin{array}{llllllllllllllllllllllllll}1 & 1 & 1 & 1 & 1 & 1 & 1 & 1 & 1 & 1 & 1 & 1 & 1 & 1 & 1 & 1 & 1 & 1 & 1 & 1 & 1 & 1 & 1 & 1 & 1 & 1\end{array}$

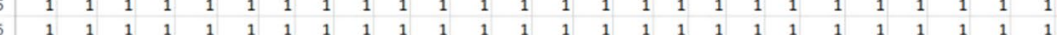
$\begin{array}{llllllllllllllllllllllllll}1 & 1 & 1 & 1 & 1 & 1 & 1 & 1 & 1 & 1 & 1 & 1 & 1 & 1 & 1 & 1 & 1 & 1 & 1 & 1 & 1 & 1 & 1 & 1 & 1 & 1\end{array}$ \begin{tabular}{l|llll|l|l|l|l|l|l|l|l|l|l|lllllllllllll}
8 & 1 & 1 & 1 & 1 & 37 & 1 & 1 & 1 & 26 & 1 & 1 & 1 & 1 & 1 & 1 & 1 & 1 & 1 & 1 & 1 & 1 & 1 & 1 & 1 & 1 & 1 \\
\hline & 1 & 1 & 1 & 23 & 35 & 43 & 1 & 27 & 26 & 1 & 1 & 29 & 1 & 1 & 1 & 1 & 1 & 1 & 1 & 1 & 1 & 1 & 1 & 1 & 1 & 1
\end{tabular}

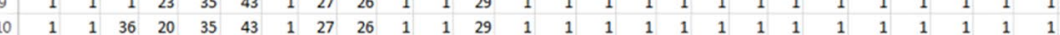
$\begin{array}{lllllllllllllllllllllllllll}11 & 1 & 1 & 36 & 28 & 35 & 43 & 1 & 33 & 26 & 1 & 1 & 20 & 1 & 1 & 45 & 19 & 1 & 1 & 1 & 1 & 1 & 1 & 1 & 1 & 1 & 1\end{array}$ $\begin{array}{llllllllllllllllllllllllll}1 & 1 & 36 & 28 & 35 & 43 & 1 & 33 & 26 & 1 & 1 & 20 & 1 & 1 & 45 & 19 & 1 & 1 & 1 & 1 & 1 & 1 & 1 & 1 & 1 & 1\end{array}$ $\begin{array}{lllllllllllllllllllllllllll}1 & 1 & 36 & 28 & 35 & 43 & 1 & 33 & 26 & 1 & 1 & 20 & 1 & 1 & 45 & 19 & 1 & 1 & 1 & 1 & 32 & 33 & 35 & 36 & 28 & 18\end{array}$ $\begin{array}{llllllllllllllllllllllllllll}14 & 1 & 1 & 36 & 28 & 35 & 43 & 1 & 33 & 26 & 1 & 1 & 20 & 1 & 1 & 39 & 19 & 1 & 1 & 1 & 1 & 25 & 35 & 41 & 36 & 33 & 18\end{array}$ $\begin{array}{llllllllllllllllllllllllllllll}15 & 1 & 1 & 36 & 28 & 35 & 43 & 1 & 33 & 26 & 1 & 1 & 20 & 1 & 1 & 39 & 19 & 1 & 1 & 1 & 1 & 25 & 27 & 41 & 36 & 33 & 26\end{array}$ $\begin{array}{lllllllllllllllllllllllllll}16 & 1 & 1 & 36 & 28 & 35 & 43 & 1 & 33 & 26 & 1 & 1 & 20 & 1 & 1 & 39 & 19 & 1 & 1 & 1 & 1 & 21 & 27 & 41 & 36 & 51 & 41\end{array}$ $\begin{array}{lllllllllllllllllllllllllllll}17 & 1 & 37 & 36 & 20 & 35 & 40 & 1 & 41 & 26 & 1 & 40 & 20 & 1 & 1 & 39 & 19 & 1 & 1 & 1 & 1 & 21 & 26 & 41 & 36 & 53 & 43\end{array}$ $\begin{array}{llllllllllllllllllllllllll}1 & 36 & 36 & 28 & 37 & 34 & 49 & 31 & 26 & 1 & 40 & 20 & 24 & 1 & 39 & 19 & 1 & 1 & 1 & 1 & 21 & 26 & 41 & 36 & 53 & 43\end{array}$ $\begin{array}{llllllllllllllllllllllllllll}9 & 1 & 36 & 36 & 28 & 37 & 34 & 49 & 33 & 12 & 1 & 40 & 20 & 36 & 1 & 39 & 19 & 1 & 1 & 1 & 1 & 21 & 25 & 41 & 35 & 54 & 44\end{array}$ $\begin{array}{llllllllllllllllllllllllllll}20 & 1 & 36 & 36 & 28 & 37 & 34 & 49 & 33 & 12 & 1 & 40 & 20 & 36 & 1 & 39 & 19 & 1 & 1 & 1 & 1 & 21 & 19 & 35 & 36 & 42 & 71\end{array}$ $\begin{array}{llllllllllllllllllllllllllllll}1 & 15 & 36 & 36 & 28 & 37 & 34 & 49 & 31 & 14 & 1 & 40 & 20 & 36 & 1 & 39 & 19 & 1 & 1 & 1 & 8 & 22 & 24 & 37 & 38 & 53 & 68\end{array}$ $\begin{array}{lllllllllllllllllllllllllllllll}22 & 15 & 36 & 36 & 28 & 37 & 34 & 49 & 31 & 14 & 1 & 40 & 20 & 36 & 1 & 39 & 8 & 1 & 18 & 1 & 11 & 21 & 22 & 51 & 51 & 85 & 71\end{array}$ \begin{tabular}{lllllllllllllllllllllllllllllll}
23 & 15 & 36 & 36 & 28 & 37 & 34 & 49 & 33 & 12 & 1 & 31 & 20 & 36 & 1 & 39 & 8 & 13 & 17 & 8 & 7 & 16 & 21 & 59 & 90 & 77 & 85 \\
\hline
\end{tabular} \begin{tabular}{lllllllllllllllllllllllllllllll}
33 & 36 & 36 & 28 & 35 & 36 & 49 & 33 & 24 & 1 & 31 & 20 & 24 & 1 & 39 & 8 & 11 & 17 & 6 & 6 & 21 & 27 & 66 & 96 & 81 & 83 \\
\hline
\end{tabular} $\begin{array}{lllllllllllllllllllllllllllllll}25 & 34 & 36 & 36 & 28 & 35 & 36 & 49 & 33 & 24 & 1 & 31 & 20 & 24 & 1 & 39 & 8 & 12 & 17 & 4 & 7 & 17 & 34 & 72 & 98 & 85 & 87 \\ \ldots\end{array}$ \begin{tabular}{l|lllllllllllllllllllllllllll}
1575 & 166 & 92 & 89 & 30 & 47 & 35 & 117 & 52 & 16 & 73 & 83 & 43 & 36 & 27 & 95 & 10 & 85 & 13 & 35 & 41 & 10 & 30 & 97 & 67 & 43 & 53
\end{tabular} (d) 
4Fig. 7 a Clusters of connected neurons generated by FC3 and FC4 channels in two SNNcubes trained on EEG data of healthy versus OP subjects in GO task; $\mathbf{b}$ the strongest connected neurons in 26 clusters; c $2 \mathrm{D}$ visualisations of $\mathbf{b}$; d static information of the dynamic cluster creation, The EEG time points are represented as rows and cluster centres are represented as columns. Each cell represents the number of neurons that belong to the cluster at this time of the learning process in the SNNcube of the spatially located neurons in a trained SNNcube to activate an output neuron; (2) the temporal order in which neurons in the SNNcube spike to activate the output neuron.

The proposed clustering methods were applied to a case study of EEG data that measured the brain activity during a cognitive task performed by three groups of subjects. (a) Clusters of connections between SNNcube and an output neuron

$\mathrm{H}$

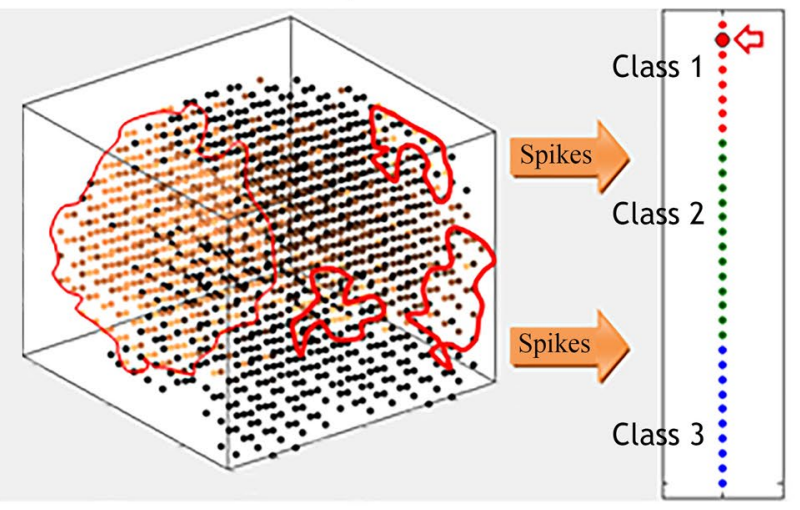

M
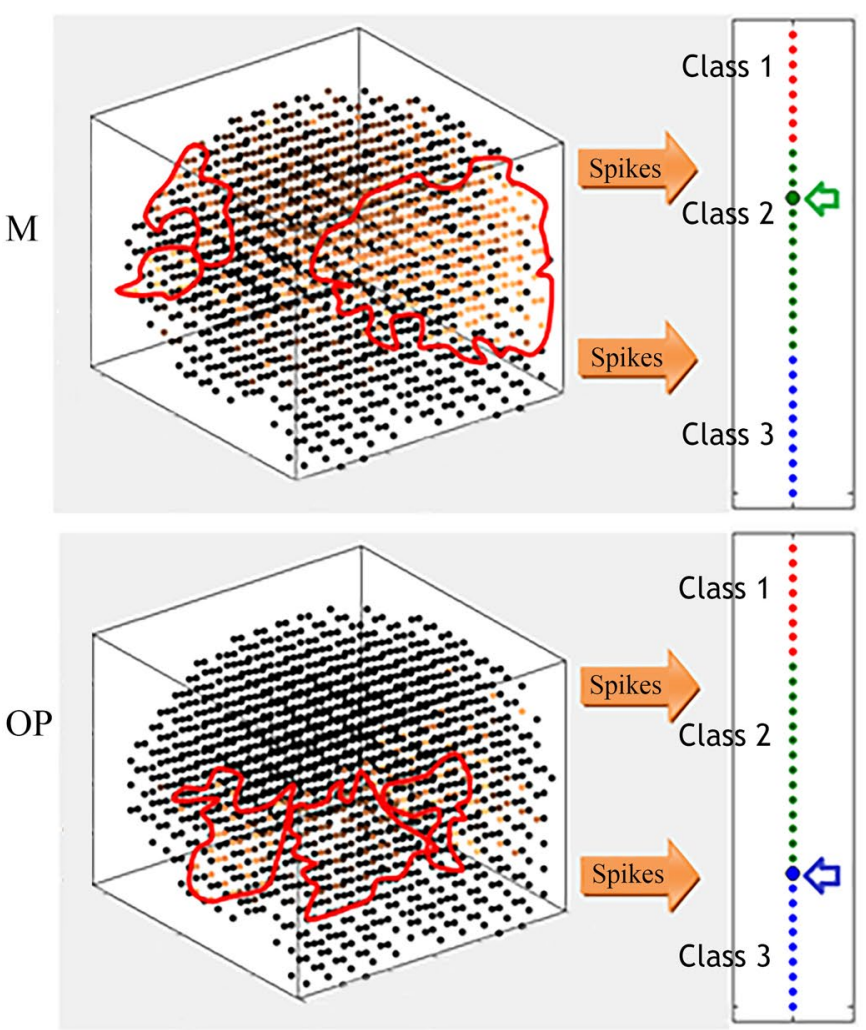

Fig. 8 In the output layer, for each training sample, one output neuron is evolved and connected to the neurons of the trained SNNcube. The output neurons are labelled by their class information in different colours (Class 1: healthy subjects is red, Class 2: M subjects in green, and Class 3: OP subjects in blue). a Personalised clusters of three selected subjects, belonging to each of the three groups $\mathrm{H}, \mathrm{M}$, (b) Clusters of spiking time in SNNcube to activate an output neuron
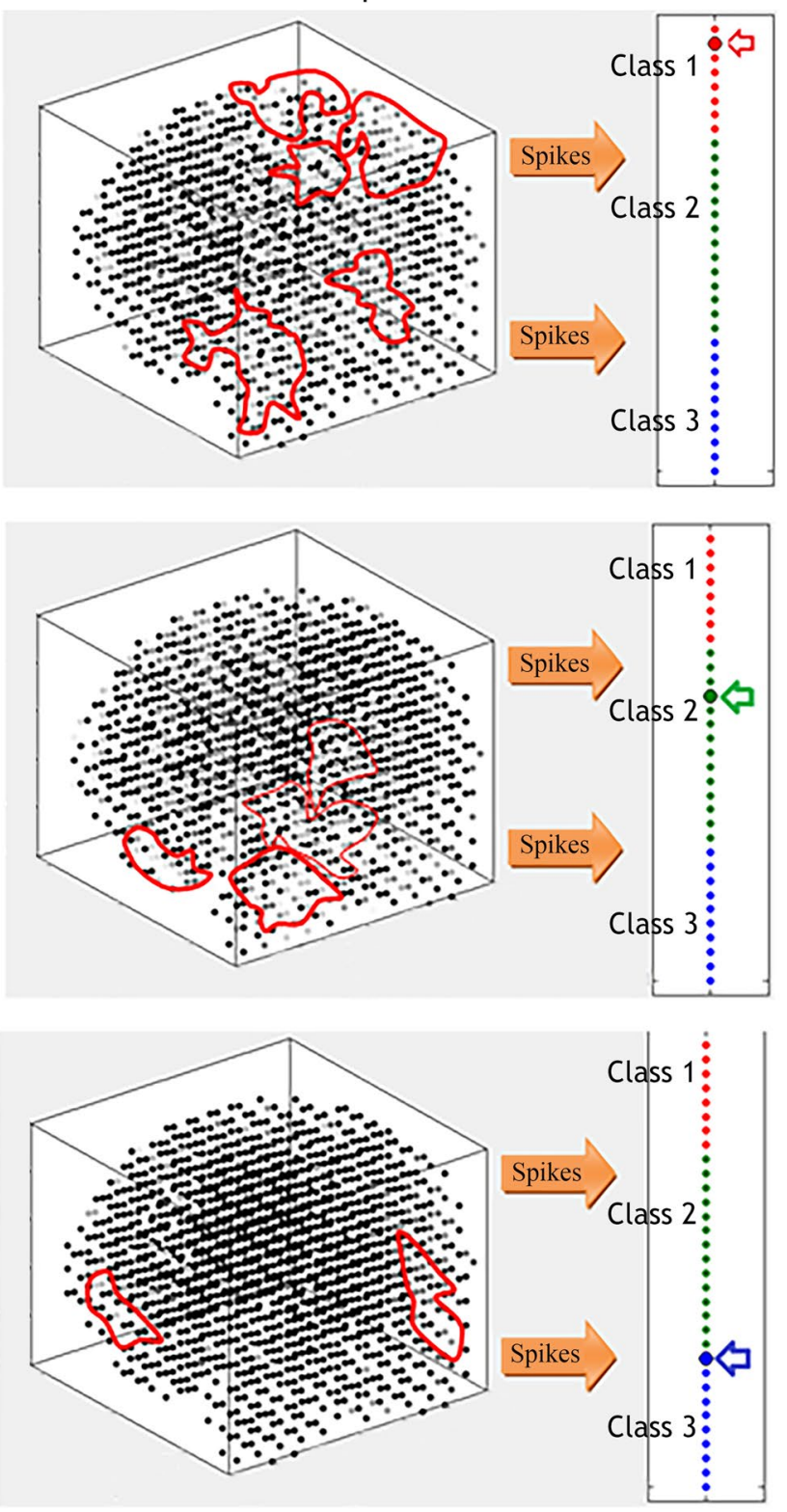

OP. Brighter neurons mean larger connection between neuron $i$ from SNNcube to the output neuron $j$. b Personalised clusters represent the similar temporal order in which the neurons in SNNcube spike to activate the corresponding output neuron. The brighter the colour of the neurons, the earlier they spike 


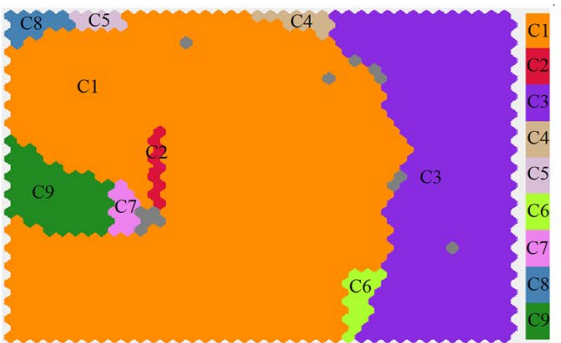

SOM trained with EEG data from $21 \mathrm{H}$ samples

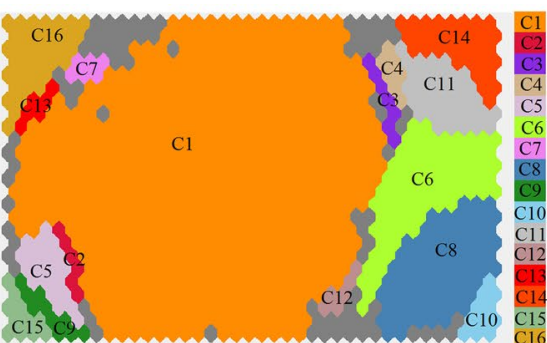

SOM trained with EEG data from 29 MMT samples

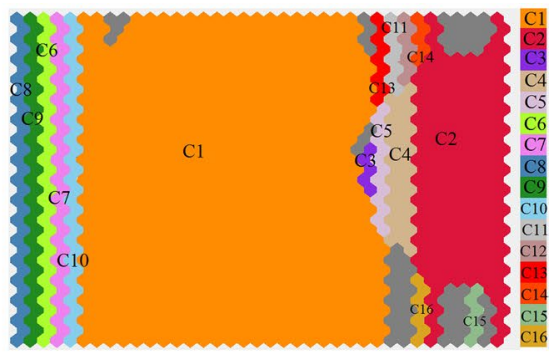

SOM trained with EEG data from 18 OP samples

Fig. 9 SOM map generated from EEG data of 21 healthy subjects, $29 \mathrm{M}$ subjects, and 18 OP subjects in a GO task. Similar healthy individual samples are clustered into nine clusters. Similar M and OP individual samples are clustered into 16 clusters each. Clusters rep-

Formation of the dynamic clusters of SSTD revealed the activity of the corresponding EEG channels at each time $t$ of the SNNcube learning. The time of cluster creation and the evolution of the cluster size were found to be meaningful in terms of EEG channel activity over time. Through tracing the sequential spikes in the SNNcube, a direct relation between the cluster creation sequence and the consecutively activated brain areas can be revealed. Personalised clusters of spiking activities in the trained SNNcube revealed temporal patterns as trajectories of functional brain activities. The proposed clustering methods enabled us to comparatively analyse the EEG data recorded from different subject groups. We found greater clusters created in frontal areas on healthy group in NOGO task. It represents that inhibition responses of the healthy group were stronger in comparison with the M and OP groups.

As a future work, we will develop an integrated clustering of both static and spatiotemporal data for personalised modelling.

Acknowledgements The research is supported by the Knowledge Engineering and Discovery Research Institute of the Auckland University of Technology (http://www.kedri.aut.ac.nz). M. GD was also supported by a summer research grant from the faculty of Design and Creative Technology of AUT. The authors would like to acknowledge Professor Robert Kydd and Dr. Bruce Russell from the University of Auckland and Dr. Grace Wang from AUT for providing us with the EEG data. We also acknowledge the assistance of Joyce D'Mello, Dr. Enmei Tu, Dr. Elisa Capecci, Lei Zhou, Israel Espinosa Ramos and Akshay Gollahalli. We are indebted to the reviewers for their detailed, precise and constructive comments and suggestions that helped us tremendously. The NeuCube software along data are available free at http://www.kedri.aut.ac.nz/neucube.

\section{References}

Abbott LF (1999) Lapicque's introduction of the integrate-and-fire model neuron (1907). Brain Res Bull 50:303-304

Bezdek JC, Ehrlich R, Full W (1984) FCM: the fuzzy c-means clustering algorithm. Comput Geosci 10:191-203 resent similarity between subjects across all channels and the whole time period, but not the dynamic spatiotemporal differences. No new information can be revealed about the dynamic processes captured in the EEG data for specific individuals or groups of individuals

Capecci E, Kasabov N, Wang GY (2015) Analysis of connectivity in NeuCube spiking neural network models trained on EEG data for the understanding of functional changes in the brain: a case study on opiate dependence treatment. Neural Netw 68:62-77

Carpenter GA, Grossberg S (1987) A massively parallel architecture for a self-organizing neural pattern recognition machine. Comput Vis Gr Image Process 37:54-115

Carter R (2014) The human brain book. Penguin books

Deboeck G, Kohonen T (1998) Visual explorations in finance: with self-organizing maps. Springer

Delbruck T (2007) jAER open source project. http://sourceforge. net/p/jaer/wiki/Home/. Accessed 15 May 2016

Deng D, Kasabov N (2000) ESOM: an algorithm to evolve self-organizing maps from on-line data streams. IJCNN 6:3-8

Deng M, Liu Q, Wang J, Shi Y (2013) A general method of spatiotemporal clustering analysis. Sci China Inf Sci 56:1-14

Ester M, Kriegel H-P, Sander J, Xu X (1996) A density-based algorithm for discovering clusters in large spatial databases with noise. In: Kdd 96(34):226-231

Gholami Doborjeh M, Kasabov N (2015) Dynamic 3D clustering of spatio-temporal brain data in the NeuCube spiking neural network architecture on a case study of fMRI data. In: ICONIP, pp 191-198

Gholami Doborjeh M, Kasabov N (2016) Personalised modelling on integrated clinical and EEG spatio-temporal brain data in the NeuCube spiking neural network system. In: WCCI: IJCNN, pp 1373-1378

Gholami Doborjeh M, Wang GY, Kasabov N, Kydd R, Russell B (2016) A spiking neural network methodology and system for learning and comparative analysis of EEG data from healthy versus addiction treated versus addiction not treated subjects. IEEE Trans Biomed Eng 63(9):1830-1841

Hartigan JA, Wong M (1979) Algorithm AS 136: a k-means clustering algorithm. J R Stat Soc Ser C Appl Stat 28:100-108

Hassinen P (2015) SOM analyzer. http://som-analyzer.software. informer.com/. Accessed 15 May 2016

Hebb DO (1949) The organization of behavior: a neuropsychological approach. Wiley

Indiveri $\mathrm{G}$ et al (2011) Neuromorphic silicon neuron circuits. Front Neurosci 5:1-23, Article 73

Johnson SC (1967) Hierarchical clustering schemes. Psychometrika $32: 241-254$

Kasabov N (2001) Evolving fuzzy neural networks for supervised/ unsupervised online knowledge-based learning. IEEE Trans Syst Man Cybern Part B Cybern 31:902-918

Kasabov N (2007) Evolving connectionist systems. Springer

\begin{tabular}{|l|l|l|l|l|}
\hline Journal : Large 12530 & Article No : 9178 & Pages : 17 & MS Code : EVOS-D-16-00033 & Dispatch : 27-3-2017 \\
\hline
\end{tabular}


Kasabov N (2012) NeuCube EvoSpike architecture for spatio-temporal. In: Artificial neural networks in pattern recognition, lecture notes in computer science. Springer, pp 225-243

Kasabov N (2014) NeuCube: a spiking neural network architecture for mapping, learning and understanding of spatio-temporal brain data. Neural Netw 52:62-76

Kasabov N, Capecci E (2015) Spiking neural network methodology for modelling, classification and understanding of EEG spatio-temporal data measuring cognitive processes. Inf Sci 294:565-575

Kasabov N, Song Q (2002) DENFIS: dynamic evolving neural-fuzzy inference system and its application for time-series prediction. IEEE Trans Fuzzy Syst 10:144-154

Kasabov N, Dhoble K, Nuntalid N, Indiveri G (2013) Dynamic evolving spiking neural networks for on-line spatio-and spectro-temporal pattern recognition. Neural Netw 41:188-201

Kasabov N, Scott NM, Tu E, Marks S (2016) Evolving spatio-temporal data machines based on the NeuCube neuromorphic framework: design methodology and selected applications. Neural Netw 78:1-14

Katwal SB, Gore JC, Marois R, Rogers BP (2013) Unsupervised spatiotemporal analysis of FMRI data using graph-based visualizations of self-organizing maps. IEEE Trans Biomed Eng 60:2472-2483

Koessler L, Maillard L, Benhadid A, Vignal JP, Felblinger J, Vespignani H, Braun M (2009) Automated cortical projection of EEG sensors: anatomical correlation via the international 10-10 system. Neuroimage 46:64-72

Kohonen T (1998) The self-organizing map. Neurocomputing 21:1-6 Lancaster JL et al (2000) Automated Talairach atlas labels for functional brain. Hum Brain Mapp 10:120-131

Lancaster JL et al (2007) Bias between MNI and Talairach coordinates analyzed using the ICBM-152 brain template. Hum Brain Mapp 28:1194-1205

Liao W, Chen H, Yang Q, Lei X (2008) Analysis of fMRI data using improved self-organizing mapping and spatio-temporal metric hierarchical clustering. IEEE Trans Med Imaging 27:1472-1483

Maass W, Thomas N, Henry M (2002) Real-time computing without stable states: a new framework for neural computation based on perturbations. Neural Comput 14:2531-2560
Mirkin B (1998) Mathematical classification and clustering. Springer

Niedermeyer E, da Silva FL (2005) Electroencephalography: basic principles, clinical applications, and related fields. Lippincott Williams and Wilkins

Ogawa S, Tank DW, Menon R, Ellermann JM, Kim SG, Merkle H, gurbil K (1992) Intrinsic signal changes accompanying sensory stimulation: functional brain mapping with magnetic resonance imaging. Proc Natl Acad Sci 89:5951-5955

Schliebs S, Fiasche M (2012) Constructing robust liquid state machines to process highly variable data streams. In: International Conference on Artificial Neural Networks, Springer, pp 604-611

Schliebs S, Capecci E, Kasabov N (2013) Spiking neural network for on-line cognitive activity classification based on EEG data. In: International Conference on Neural Information Processing, Springer, pp 55-62

Song S, Miller KD, Abbott LF (2000) Competitive Hebbian learning through spike-timing-dependent synaptic plasticity. Nat Neurosci 3:919-926

Talairach J, Tournoux P (1988) Co-planar stereotaxic atlas of the human brain. 3-Dimensional proportional system: an approach to cerebral imaging. Thieme Medical Publishers, New York

Tanay A, Sharan R, Shamir R (2002) Discovering statistically significant biclusters in gene expression data. Bioinformatics 18:136-144

Thorpe S, Gautrais J (1998) Rank order coding. In: Computational neuroscience, Springer, pp 113-118

Tu E, Cao L, Yang J, Kasabov N (2014) A novel graph-based k-means for nonlinear manifold clustering and representative selection. Neurocomputing 143:109-122

Tu E, Kasabov N, Yang J (2016) Mapping temporal variables into the NeuCube for improved pattern recognition, predictive modelling and understanding of stream data. IEEE Trans Neural Netw Learn Syst. doi:10.1109/TNNLS.2016.2536742

Wang W, Yang J, Muntz R (1997) STING: a statistical information grid approach to spatial data mining. VLDB 97:186-195

Zhou D, Bousquet O, Lal TN, Weston J (2004) Learning with local and global consistency. Adv Neural Inf Process Syst 16:321-328

\begin{tabular}{|l|l|l|l|l|}
\hline Journal : Large 12530 & Article No : 9178 & Pages : 17 & MS Code : EVOS-D-16-00033 & Dispatch : 27-3-2017 \\
\hline
\end{tabular}

